\title{
Positroid stratification of orthogonal Grassmannian and ABJM amplitudes
}

\author{
Joonho $\mathrm{Kim}^{a}$ and Sangmin Lee $\mathrm{Le}^{a, b, c, d}$ \\ ${ }^{a}$ Department of Physics and Astronomy, Seoul National University, \\ 1 Gwanakro Gwanak-gu, Seoul 151-747, Korea \\ ${ }^{b}$ Center for Theoretical Physics, Seoul National University, \\ 1 Gwanakro Gwanak-gu, Seoul 151-747, Korea \\ ${ }^{c}$ College of Liberal Studies, Seoul National University, \\ 1 Gwanakro Gwanak-gu, Seoul 151-742, Korea \\ ${ }^{d}$ School of Physics, Korea Institute for Advanced Study, \\ 85 Hoegiro Dongdaemun-gu, Seoul 130-722, Korea \\ E-mail: joonho0@gmail.com, sangmin@snu.ac.kr
}

\begin{abstract}
A novel understanding of scattering amplitudes in terms of on-shell diagrams and positive Grassmannian has been recently established for four dimensional Yang-Mills theories and three dimensional Chern-Simons theories of ABJM type. We give a detailed construction of the positroid stratification of orthogonal Grassmannian relevant for ABJM amplitudes. On-shell diagrams are classified by pairing of external particles. We introduce a combinatorial aid called 'OG tableaux' and map each equivalence class of on-shell diagrams to a unique tableau. The on-shell diagrams related to each other through BCFW bridging are naturally grouped by the OG tableaux. Introducing suitably ordered BCFW bridges and positive coordinates, we construct the complete coordinate charts to cover the entire positive orthogonal Grassmannian for arbitrary number of external particles. The graded counting of OG tableaux suggests that the positive orthogonal Grassmannian constitutes a combinatorial polytope.
\end{abstract}

Keywords: Field Theories in Lower Dimensions, Scattering Amplitudes, Extended Supersymmetry, Differential and Algebraic Geometry

ARXIV EPRINT: 1402.1119 


\section{Contents}

1 Introduction and conclusion $\quad 1$

2 On-shell diagrams for ABJM amplitudes 4

2.1 Elements of on-shell diagrammatics 4

$\begin{array}{lll}2.2 & \text { BCFW bridge } & 6\end{array}$

2.3 Amalgamation and permutation 9

3 Reality and positivity of orthogonal Grassmannian $\quad 11$

3.1 Complex OG 11

$\begin{array}{lll}3.2 & \text { Reality and positivity of OG } & 12\end{array}$

$\begin{array}{lll}3.3 & \text { Conversion rule } & 15\end{array}$

4 Positroid stratification $\quad 16$

$\begin{array}{ll}4.1 \text { OG tableaux } & 16\end{array}$

$\begin{array}{ll}4.2 \text { Canonically positive coordinates } & 19\end{array}$

4.3 Polytope 24

\section{Introduction and conclusion}

The study of scattering amplitudes in gauge theories has gone through many stages of exciting developments (see [1] for an up-to-date review). A common theme in many recent breakthroughs is reformulation of the gauge theory in such a way to uncover hidden structures that are hardly visible in the traditional approach.

The Grassmannian integral [2] is a relatively new reformulation notably far removed from the traditional approach. Tree amplitudes and loop integrands of a planar gauge theory are produced from a contour integral over the Grassmannian $\mathrm{G}(k, n)$, where $n$ is the total number of external particles and $k$ is the number of external particles with negative helicity. The geometric structure makes the conformal symmetry and dual conformal symmetry completely manifest while relegating locality and unitarity to an emergent property. Although the Grassmannian integral inherits some crucial features from its predecessors such as the twistor string theory $[3,4]$ and the BCFW recursion relation $[5,6]$, its physical origin had remained elusive for years.

In a remarkable paper [7], Arkani-Hamed et al. introduced the notion of 'on-shell diagrams' which in a sense provides a microscopic structure underlying the Grassmannian integral. The vertices of on-shell diagrams are the gauge invariant three-point amplitudes with $(++-)$ or $(+--)$ helicity assignments. They showed how to rewrite the vertices in terms of integrals over $\mathrm{G}(1,3)$ or $\mathrm{G}(2,3)$. By integrating out internal lines, they combine small Grassmannian integrals to form a big integral over $\mathrm{G}(k, n)$ for arbitrary $k$ and $n$. 
The BCFW deformation of momenta appears naturally in on-shell diagrams and 'bridges' two external legs with a new internal line and two vertices. While probing the structure of Grassmannian integral at a deeper level, the authors of [7] also encountered a number of modern topics in mathematics such as affine permutation, positive Grassmannian, positroid stratification, and cluster algebra. They also noted that the on-shell diagram approach can be applied to any gauge theory in four dimensions, although it takes by far the simplest form for the $\mathcal{N}=4$ super-Yang-Mills theory $\left(\mathrm{SYM}_{4}\right)$.

In three dimensions, a class of $\mathcal{N}=6$ superconformal Chern-Simons matter theories [812], widely known as ABJM theory, serves as a main testing ground for novel methods on scattering amplitudes. An initial step toward the on-shell diagram approach to ABJM theory was already taken in [7], based on the an integral over the orthogonal Grassmannian $\mathrm{OG}(k, 2 k)$ [13]. The on-shell diagrams of ABJM theory consist of a unique quartic vertex and an internal line. The permutation governing the planar diagrams is a complete pairing of the $2 k$ external particles. Two diagrams sharing the same permutation are equivalent to each other up to Yang-Baxter equivalence moves. The BCFW bridge connects two external legs by creating an extra vertex between them.

Recently, Huang and Wen [14] further studied the on-shell diagrams for ABJM amplitudes. They refined the on-shell diagrams with some sign factors to account for the two disjoint 'branches' of orthogonal Grassmannian. This is crucial since the full tree amplitudes receive contributions from both branches, as noted earlier in [15]. By rewriting the BCFW recursion relation for ABJM theory [15] in terms of on-shell diagrams, they constructed on-shell diagram representation of all tree-level amplitudes. They also introduced a set of coordinates in which all consecutive minors take a simple form. Finally, they defined the notion of positive orthogonal Grassmannian. In contrast to the ordinary Grassmannian $\mathrm{G}(k, n)$, it is slightly non-trivial to define reality conditions on $\mathrm{OG}(k, 2 k)$.

The aim of this paper is to cover a topic that was notably missing in $[7,14]$. Given a permutation, it is desirable to select a particular representative of the equivalence class of on-shell diagrams, and assign canonically positive coordinates to it. The construction of canonical coordinates elucidates how the Grassmannian integral is decomposed into a series of BCFW bridging. The canonical coordinates also enjoy several nice properties; for instance, the measure of the Grassmannian integral takes a simple ' $d(\log )$ ' form, and the boundaries of the positive orthogonal Grassmannian become zero loci of the coordinates. This problem of assigning canonical coordinates was solved completely in four dimensions in [7] using a relation to the mathematics of 'positroid stratification' [16, 17]. We will solve the problem in three dimensions by introducing a combinatorial device similar to those of [16], which we call 'OG tableaux'. To our knowledge, mathematical studies of positive orthogonal Grassmannian remain incomplete and not readily accessible to physicists. See, e.g., [18] for a related work.

The rest of this paper is organized as follows. Section 2 is a brief review of what is known for on-shell diagrams for ABJM theory from earlier works [7, 14]. The elementary 4-particle vertex can be understood both as a BCFW bridge and an integral over OG $(2,4)$. Integration over internal lines 'amalgamates' copies of $\mathrm{OG}(2,4)$ and builds up bigger $\mathrm{OG}(k, 2 k)$. In section 3 , we take a closer look at the geometry of orthogonal Grass- 
mannian. We examine real slices of $\mathrm{OG}(k, 2 k)$ and discuss how to define positivity on a real slice. The real version of the BCFW bridge $\mathrm{OG}(2,4)$ and amalgamation naturally suggests how to introduce manifestly real coordinates on the orthogonal Grassmannian. Section 4 reports the main results of this paper. We begin with mapping the on-shell diagrams to what we call 'OG tableaux'. The tableau notation serves several purposes. It fixes the ambiguity coming from Yang-Baxter equivalence relation in a canonical way. That helps us to count distinct on-shell diagrams, which are interpreted geometrically as cells of the positive Grassmannian. Moreover, the OG tableaux can be used to assign 'canonical positive coordinates' for all on-shell diagrams. The integration measure in the canonical coordinates is a product of simple $d \log$ factors. The OG tableaux also help us to study mathematics of $\mathrm{OG}_{+}(k, 2 k)$. The positive Grassmannian $\mathrm{G}_{+}(k, n)$ is known to form a combinatorial polytope called 'Eulerian poset' [19]. The graded counting of OG tableaux suggests that the positive orthogonal Grassmannian $\mathrm{OG}_{+}(k, 2 k)$ similarly defines an Eulerian poset at each $k$. Geometrically, the graded counting hints at the possibility that $\mathrm{OG}_{+}(k, 2 k)$ may have a topology of a ball. We verify this conjecture for $k=2,3$ and leave the generalization to higher $k$ as an open problem.

This paper focuses on a formal aspect of orthogonal Grassmannian and makes little direct contributions to ABJM amplitudes. However, the results of this paper clearly opens up a few directions of further research. Here, we list three prominent possibilities.

First, the issue of Yangian symmetry, which unites the ordinary and dual superconformal symmetries, could be revisited. While there are strong evidences for the Yangian symmetry of ABJM amplitudes [13, 15, 20-22], a formulation with manifest dual superconformal symmetry has not been found. For SYM 4 , such a dual formulation was found earlier [23, 24] and laid a foundation for further discoveries such as the 'amplituhedron' [25]. In [7], the Yangian symmetry was interpreted geometrically as diffeomorphisms which leave the measure on $\mathrm{G}_{+}(k, n)$ invariant. A similar interpretation for $\mathrm{OG}_{+}(k, 2 k)$, if possible, would shed new light on the Yangian symmetry of ABJM amplitudes.

Second, our work could be related to twistor string models for ABJM amplitudes. For tree-level amplitudes of $\mathrm{SYM}_{4}$, the equivalence between the twistor string formula [4] and the Grassmannian formula [2] was established in [26]. Simply put, the derivation consists of three steps: specifying the integration contour of the Grassmannian integral, deforming its integrand with no loss of residues, and integrating out some variables. Along the same line of reasoning, a twistor string formula was proposed in [27] (and recently rederived from different viewpoints in $[28,29]$ ), but the derivation was less solid due to limited understanding of the integration contours. The canonical coordinates defined in this paper could be useful in finding a refined derivation comparable to that of [26].

Third, the formal structure of the positive orthogonal Grassmannian could be probed at a deeper level. To define the canonical coordinate system, we suppressed the YangBaxter equivalence moves in a particular 'frame'. If we move to another frame, the new coordinates would be related to the old ones in a non-trivial way. For $\mathrm{SYM}_{4}$, the coordinate transformation has an intriguing connection to the mathematics of cluster algebra [30,31]. It would be interesting to figure out the ABJM counterpart of the story. See a recent work by Huang, Wen and Xie [32] for a related discussion. 
We conclude this introduction with some shorthand notations. We will write $(\mathrm{P}) \mathrm{OG}$ for (positive) orthogonal Grassmannian, and abbreviate $\mathrm{OG}(k, 2 k)$ and $\mathrm{OG}_{+}(k, 2 k)$ by $\mathrm{OG}_{k}$ and $\mathrm{POG}_{k}$, respectively.

Note added. While an early version of this paper was being revised for publication, we received a preprint by Lam [38] which rigorously prove a mathematical theorem stating that $\mathrm{POG}_{k}$ defines an Eulerian poset for all $k$.

\section{On-shell diagrams for ABJM amplitudes}

In this section, we review some salient features of the on-shell diagrams for ABJM amplitudes $[7,14]$. We first recall the kinematics of the ABJM amplitudes and the definition of the OG integral to set the stage for the on-shell diagram. After introducing the fundamental building blocks, a quartic vertex and an internal line, we examine two ways to construct the most general on-shell diagrams. One is BCFW bridging, which enables us to add a vertex one at a time to a given diagram. The other is amalgamation, which merges two diagrams into a larger one by integrating over an internal line. For both methods, we explain how the diagrammatics is reflected in the OG integral, thereby making the microscopic decomposition of OG integral manifest. Finally, we comment briefly on the 'Yang-Baxter' equivalence relation for different diagrams corresponding to the same amplitude as well as the bubble diagrams.

\subsection{Elements of on-shell diagrammatics}

Kinematics. The ABJM theory is a Chern-Simons-matter gauge theory in three dimensions with $\mathcal{N}=6$ superconformal symmetry. The symmetry group is $\operatorname{OSp}(6 \mid 4)$ whose bosonic part contains $\operatorname{Sp}(4, \mathbb{R}) \simeq \operatorname{Spin}(2,3)$ conformal symmetry and $\mathrm{SO}(6) R$-symmetry. As shown in [20], the $\operatorname{OSp}(6 \mid 4)$ symmetry becomes manifest if we work in a superspace $\Lambda=\left(\lambda^{\alpha} ; \eta^{I}\right) \in \mathbb{C}^{2 \mid 3}$.

In this representation, the superconformal generators come in three types:

$$
\Lambda \frac{\partial}{\partial \Lambda}, \quad \Lambda \Lambda, \quad \frac{\partial^{2}}{\partial \Lambda \partial \Lambda} .
$$

The superspace notation will guarantee the invariance of the amplitudes under the $(\Lambda \partial / \partial \Lambda)$ generators. Let us decompose the $(\Lambda \Lambda)$ generators,

$$
p^{\alpha \beta}=\lambda^{\alpha} \lambda^{\beta}, \quad q^{\alpha I}=\lambda^{\alpha} \eta^{I}, \quad r^{I J}=\eta^{I} \eta^{J} .
$$

In a scattering process, the invariance under $p^{\alpha \beta}$ and $q^{\alpha I}$ can be imposed by the supermomentum conserving delta functions

$$
\delta^{3}(P) \delta^{6}(Q) \quad \text { with } \quad P:=\sum_{i} p_{i}^{\alpha \beta}, \quad Q:=\sum_{i} q_{i}^{\alpha I} .
$$

The $r^{I J}$ invariance introduces a coset $\mathrm{O}(2 k-4) / \mathrm{U}(k-2)$ for the $2 k$-point amplitude [20]. The coset structure was a precursor to the OG integral for ABJM theory [13]. Once the 
invariance under the $(\Lambda \Lambda)$ generators is confirmed, the invariance under the remaining $\left(\partial^{2} / \partial \Lambda \partial \Lambda\right)$ generators will follow from the superconformal inversion, which acts on $\Lambda$ as Fourier transformation $(\Lambda \leftrightarrow \partial / \partial \Lambda)$.

The spinors $\lambda_{i}$ are contracted via the invariant tensor $\epsilon_{\alpha \beta}\left(\epsilon_{12}=-\epsilon^{12}=1\right)$ of $\operatorname{SL}(2, \mathbb{R}) \simeq \operatorname{Spin}(1,2)$ Lorentz group,

$$
\langle i j\rangle:=\lambda_{i}^{\alpha} \lambda_{j \alpha}=\lambda_{i}^{\alpha} \epsilon_{\alpha \beta} \lambda_{j}^{\beta} .
$$

The ABJM theory contains two matter multiplets with opposite gauge charge. The particle/anti-particle superfields take the form

$$
\begin{aligned}
& \Phi=\phi^{4}+\eta^{I} \psi_{I}+\frac{1}{2} \epsilon_{I J K} \eta^{I} \eta^{J} \phi^{K}+\frac{1}{6} \epsilon_{I J K} \eta^{I} \eta^{J} \eta^{K} \psi_{4}, \\
& \bar{\Phi}=\bar{\psi}^{4}+\eta^{I} \bar{\phi}_{I}+\frac{1}{2} \epsilon_{I J K} \eta^{I} \eta^{J} \bar{\psi}^{K}+\frac{1}{6} \epsilon_{I J K} \eta^{I} \eta^{J} \eta^{K} \bar{\phi}_{4} .
\end{aligned}
$$

The color-ordered tree-level super-amplitudes, $A_{2 k}\left(\Lambda_{1}, \cdots, \Lambda_{2 k}\right)$, are functions of $\Lambda_{i}$. Following the convention of $[13,15]$, we choose to associate $\Lambda_{\text {odd/even }}$ to $\bar{\Phi} / \Phi$ multiplet. As noted in [20], the kinematics and the multiplet structure imply the ' $\Lambda$-parity',

$$
A_{2 k}\left(\Lambda_{1}, \cdots,-\Lambda_{i}, \cdots, \Lambda_{2 k}\right)=(-1)^{i} A_{2 k}\left(\Lambda_{1}, \ldots, \Lambda_{i}, \cdots, \Lambda_{2 k}\right) .
$$

and symmetry under the cyclic shift by two sites,

$$
A\left(\Lambda_{1}, \Lambda_{2}, \cdots, \Lambda_{2 k}\right)=(-1)^{k} A\left(\Lambda_{3}, \Lambda_{4}, \cdots, \Lambda_{2 k}, \Lambda_{1}, \Lambda_{2}\right) .
$$

OG integral. A central object of interest in this paper is the OG integral [13]:

$$
\mathcal{L}_{2 k}(\Lambda)=\int \frac{d^{k \times 2 k} C}{\operatorname{vol}[\mathrm{GL}(k)]} \frac{\delta^{k(k+1) / 2}\left(C \cdot C^{T}\right) \delta^{2 k \mid 3 k}(C \cdot \Lambda)}{M_{1} M_{2} \cdots M_{k-1} M_{k}} .
$$

The integration variable $C$ is a $(k \times 2 k)$ matrix. The dot products denote $\left(C \cdot C^{T}\right)_{m n}=$ $C_{m i} C_{n i},(C \cdot \Lambda)_{m}=C_{m i} \Lambda_{i}$. The $i$-th consecutive minor $M_{i}$ of $C$ is defined by

$$
M_{i}=\left(C_{i}, C_{i+1}, \cdots, C_{i+k-1}\right)=\epsilon^{m_{1} \cdots m_{k}} C_{m_{1}(i)} C_{m_{2}(i+1)} \cdots C_{m_{k}(i+k-1)} .
$$

In [13], this formula was conjectured to reproduce the $2 k$-point tree level amplitude upon a suitable choice of integration contour. The conjecture was verified up to $k=4$ in [15]. We will review the on-shell diagram approach to the OG integral (2.8), initiated in [7] and elaborated in [14], in a way to facilitate the introduction of the positroid stratification to be presented in section 4 .

Building blocks. The on-shell diagrams for ABJM amplitudes are planar diagrams drawn on a disk with $2 k$ boundary points representing cyclically ordered external particles. Schematically, the building blocks of the diagrams take the following form [7]:

$$
\text { Internal line: } \quad=\quad=\int d^{2} \lambda d^{3} \eta:=\int d^{2 \mid 3} \Lambda:=\int " d \Lambda ",
$$




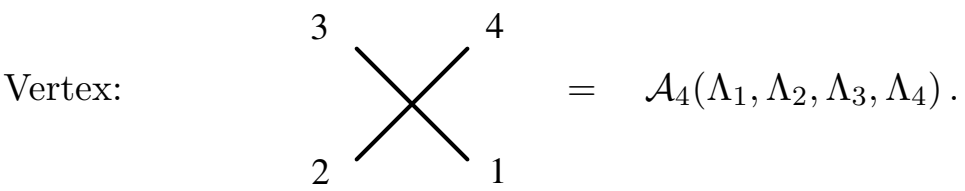

The quartic vertex is precisely the 4-point tree amplitude first computed in [33] and reproduced from the OG integral in $[13,15]$.

The graphical notations in (2.10) and (2.11) are not fully well-defined as they stand. The 4-point amplitude $A_{4}$ does not have a $\mathbb{Z}_{4}$ cyclic symmetry. Instead, it is odd under a cyclic shift by two sites,

$$
A_{4}(1,2,3,4)=-A_{4}(3,4,1,2) .
$$

Accounting for this symmetry, when the simpler notation is likely to cause confusion, we will use a refined notation for the vertex.

Vertex:

$$
2 \int_{1}^{3} 4=\frac{\delta^{3}(P) \delta^{6}(Q)}{\langle 12\rangle\langle 23\rangle} \text {. }
$$

The internal line (2.10) means that two sub-diagrams, say $F(\Lambda)$ and $G(\Lambda)$, can be 'glued' by an integral of the form,

$$
\int d \Lambda F(\Lambda) G(-i \Lambda)=\int d \Lambda_{1} d \Lambda_{2} \delta\left(i \Lambda_{1}+\Lambda_{2}\right) F\left(\Lambda_{1}\right) G\left(\Lambda_{2}\right) .
$$

The factor of $i$ reflects momentum conservation between two particles: $\lambda \lambda+(i \lambda)(i \lambda)=0$. By convention, we will assign $\Lambda_{1}$ and $\Lambda_{2}$ in (2.14) to an odd $(\bar{\Phi})$ and an even $(\Phi)$ multiplet, respectively.

\subsection{BCFW bridge}

Vertex as BCFW bridge. As shown in [7, 14], it is possible to build up complicated on-shell diagrams from a simpler one by adding vertices via 'BCFW bridges'. In this subsection, we review how to interpret the elementary vertex (2.13) as a BCFW bridge, and establish our convention for coordinates and sign factors.

We recall from [15] that the $\mathrm{BCFW}$ deformation acts as an $\mathrm{O}(2 k, \mathbb{C})$ rotation on the kinematic variables $\Lambda_{i}$, which leaves the total super-momentum invariant. For the fourparticle vertex, the total super-momentum is

$$
\begin{aligned}
& P=\lambda_{1} \lambda_{1}+\lambda_{2} \lambda_{2}+\lambda_{3} \lambda_{3}+\lambda_{4} \lambda_{4}=0, \\
& Q=\lambda_{1} \eta_{1}+\lambda_{2} \eta_{2}+\lambda_{3} \eta_{3}+\lambda_{4} \eta_{4}=0 .
\end{aligned}
$$

Moving $\lambda_{3}$ and $\lambda_{4}$ to the right-hand-side in (2.15) and squaring, we find

$$
\langle 12\rangle^{2}=\langle 34\rangle^{2} \Longrightarrow\langle 12\rangle=\sigma\langle 34\rangle \quad(\sigma= \pm 1)
$$


The sign factor $\sigma$ defines two 'branches' of kinematic configuration. As we will see shortly, the same $\sigma$ will define two branches of $\mathrm{OG}_{2}$.

To reveal the structure of the BCFW bridge, we begin with the vertex

$$
A_{4}\left(\Lambda_{1}, \Lambda_{2}, \Lambda_{3}, \Lambda_{4}\right)=\frac{\delta(P) \delta(Q)}{\langle 12\rangle\langle 23\rangle} .
$$

Inserting two identities, ${ }^{1}$

$$
1=-\langle 34\rangle \int d c_{i 3} \wedge d c_{i 4} \delta^{2}\left(\lambda_{i}+c_{i 3} \lambda_{3}+c_{i 4} \lambda_{4}\right) \quad \text { for } i=1,2
$$

changes the momentum-conserving delta function into

$$
\begin{aligned}
\delta^{3}(P) & =\delta^{3}\left(\lambda_{3} \lambda_{3}\left(1+c_{13}^{2}+c_{23}^{2}\right)+\lambda_{4} \lambda_{4}\left(1+c_{14}^{2}+c_{24}^{2}\right)+\left(\lambda_{3} \lambda_{4}+\lambda_{4} \lambda_{3}\right)\left(c_{13} c_{14}+c_{23} c_{24}\right)\right) \\
& =-\langle 34\rangle^{-3} \delta\left(1+c_{13}^{2}+c_{23}^{2}\right) \delta\left(1+c_{14}^{2}+c_{24}^{2}\right) \delta\left(c_{13} c_{14}+c_{23} c_{24}\right) .
\end{aligned}
$$

Taking the change of variables

$$
\left(\begin{array}{ll}
c_{13} & c_{14} \\
c_{23} & c_{24}
\end{array}\right)=i\left(\begin{array}{ll}
r_{3} \sin t_{3} & r_{4} \cos t_{4} \\
r_{3} \cos t_{3} & r_{4} \sin t_{4}
\end{array}\right)
$$

and integrating out $r_{3}$ and $r_{4}$, we find

$$
\begin{aligned}
A_{4}= & -\frac{\delta^{6}(Q)}{4 i\langle 34\rangle^{3}} \int \frac{d t_{3} \wedge d t_{4}}{\sin t_{4} \cos \left(t_{3}+t_{4}\right)} \delta\left(\sin \left(t_{3}+t_{4}\right)\right) \\
& \times \delta^{2}\left(\lambda_{1}+i \sin t_{3} \lambda_{3}+i \cos t_{4} \lambda_{4}\right) \delta^{2}\left(\lambda_{2}+i \cos t_{3} \lambda_{3}+i \sin t_{4} \lambda_{4}\right) \\
= & \frac{\delta^{6}(Q)}{4 i\langle 34\rangle^{3}} \int \frac{\sigma d t_{3}}{\sin t_{3}} \sum_{\sigma} \delta^{2}\left(\lambda_{1}+i s_{3} \lambda_{3}+i \sigma c_{3} \lambda_{4}\right) \delta^{2}\left(\lambda_{2}+i c_{3} \lambda_{3}-i \sigma s_{3} \lambda_{4}\right),
\end{aligned}
$$

where the first delta function is localized at $t_{3}+t_{4}=0$ or $\pi$. The fermionic delta function can be rearranged as

$$
\begin{aligned}
\delta^{6}(Q) & =-\langle 12\rangle^{-3} \delta^{3}\left(\langle 12\rangle \eta_{1}+\langle 32\rangle \eta_{3}+\langle 42\rangle \eta_{4}\right) \delta^{3}\left(\langle 21\rangle \eta_{2}+\langle 31\rangle \eta_{3}+\langle 41\rangle \eta_{4}\right) \\
& =\sigma\langle 34\rangle^{3} \delta^{3}\left(\eta_{1}+i \sin t_{3} \eta_{3}+i \sigma \cos t_{3} \eta_{4}\right) \delta^{3}\left(\eta_{2}+i \cos t_{3} \eta_{3}-i \sigma \sin t_{3} \eta_{4}\right)
\end{aligned}
$$

Collecting all the ingredients, we obtain

$$
A_{4}=\sum_{\sigma= \pm} \int \frac{d t}{4 i \sin t} \delta^{2 \mid 3}\left(\Lambda_{1}+\Lambda_{4}^{\sigma}(t)\right) \delta^{2 \mid 3}\left(\Lambda_{2}+\Lambda_{3}^{\sigma}(t)\right)
$$

where

$$
\left(\begin{array}{c}
\Lambda_{3}^{\sigma}(t) \\
\Lambda_{4}^{\sigma}(t)
\end{array}\right)=\left(\begin{array}{cc}
i \cos t & -i \sigma \sin t \\
i \sin t & i \sigma \cos t
\end{array}\right)\left(\begin{array}{l}
\Lambda_{3} \\
\Lambda_{4}
\end{array}\right) .
$$

Pictorially, as shown in figure 1(a), the result might be summarized as building a BCFW bridge between two 'free propagators' (14) and (23). During the derivation, we chose two adjacent legs $\{1,2\}$ as 'sources' and the other legs $\{3,4\}$ as 'sinks'.

\footnotetext{
${ }^{1}$ Essentially the same computation was done in [34] with particular reality conditions on $\lambda_{i}$. Here, we leave $\lambda_{i}$ as complex variables, and treat the delta-functions as analytic functions.
} 


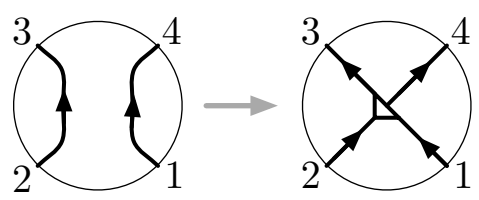

(a)

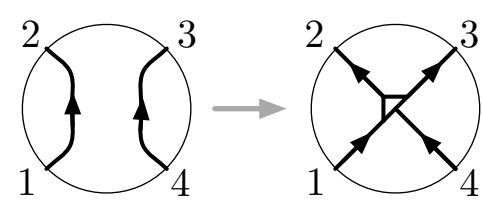

(b)

Figure 1. 4-point vertex as a BCFW bridge with two adjacent 'sources' legs.

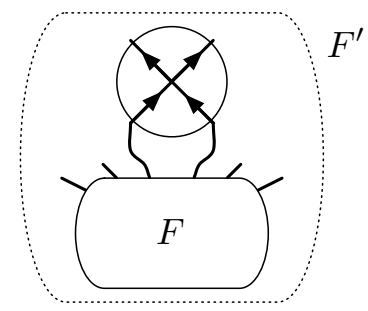

Figure 2. Adding a vertex to an on-shell diagram via BCFW bridge.

Other choices of sources and sinks are possible. Up to the cyclic symmetry (2.12), the only other possibility for adjacent source legs is $\{1,4\}$, as depicted in figure $1(\mathrm{~b})$. With the branch parameter defined by $\langle 14\rangle=\sigma\langle 23\rangle, A_{4}$ becomes

$$
A_{4}=\sum_{\sigma= \pm} \int \frac{d t}{4 i \sin t} \delta^{2 \mid 3}\left(\Lambda_{1}+\Lambda_{2}^{\sigma}(t)\right) \delta^{2 \mid 3}\left(\Lambda_{4}+\Lambda_{3}^{\sigma}(t)\right)
$$

where

$$
\left(\begin{array}{c}
\Lambda_{2}^{\sigma}(t) \\
\Lambda_{3}^{\sigma}(t)
\end{array}\right)=\left(\begin{array}{cc}
i \sigma \cos t & -i \sin t \\
-i \sigma \sin t & -i \cos t
\end{array}\right)\left(\begin{array}{c}
\Lambda_{2} \\
\Lambda_{3}
\end{array}\right) .
$$

We may also consider taking non-adjacent source legs, $\{1,3\}$ or $\{2,4\}$. They will not be used in subsequent sections, so we omit them here. Interested readers are referred to [14].

General BCFW bridging. The BCFW bridge can be used to add a vertex to an onshell diagram at a fixed number of external legs. The idea is sketched in figure 2 .

The rotation matrix, as in $(2.25)$ or $(2.27)$, is an element of an $\mathrm{O}(2, \mathbb{C})$ subgroup of $\mathrm{O}(2 k, \mathbb{C})$ acting on the kinematic variables $\Lambda_{i}$. It is worth noting whether the rotation matrix belongs to the orientation preserving $\mathrm{SO}(2 k, \mathbb{C})$ subgroup of $\mathrm{O}(2 k, \mathbb{C})$ or the orientation reversing one. In the case depicted in figure $1(\mathrm{a}), \sigma=+1$ preserves orientation. In contrast, in the case of figure $1(\mathrm{~b}), \sigma=-1$ is the one that preserves orientation.

Vertex as $\mathbf{O G}_{2}$. We proceed to relate the BCFW bridge to $\mathrm{OG}_{2}$. Consider the integral,

$$
\mathcal{L}_{4}(\Lambda)=\int \frac{d^{2 \times 4} C}{\operatorname{vol}[\mathrm{GL}(2)]} \frac{\delta^{3}\left(C \cdot C^{T}\right) \delta^{4 \mid 6}(C \cdot \Lambda)}{(12)(23)} .
$$

We will show that $\mathcal{L}_{4}$ reproduces $A_{4}$ in the form (2.24) or (2.26). For the former, depicted in figure $1(\mathrm{a})$, the choice of source legs $\{1,2\}$ naturally translates into a gauge fixing of the 
matrix $C$ with 'pivot' columns $\{1,2\}$,

$$
C=\left(\begin{array}{llll}
1 & 0 & c_{13} & c_{14} \\
0 & 1 & c_{23} & c_{24}
\end{array}\right)
$$

We can proceed in two different ways. First, as explained in [15], we can solve the bosonic part of the kinematic delta function $\delta^{4 \mid 6}(C \cdot \Lambda)$. Since there are four delta functions for four variables, for generic values of $\lambda_{i}(i=1,2,3,4)$, the solution to the delta function constraint is unique. Inserting the solution back to the integral, we arrive at the expression for $A_{4}$ in (2.18). Alternatively, we can leave $\delta^{4 \mid 6}(C \cdot \Lambda)$ aside and insert the gauge-fixed $C$ (2.29) into the orthogonality constraint delta function,

$$
\delta^{3}\left(C \cdot C^{T}\right)=\delta\left(1+c_{13}^{2}+c_{14}^{2}\right) \delta\left(1+c_{23}^{2}+c_{24}^{2}\right) \delta\left(c_{13} c_{23}+c_{14} c_{24}\right),
$$

which takes a similar form as (2.20). Taking the change of variables,

$$
\left(\begin{array}{ll}
c_{13} & c_{14} \\
c_{23} & c_{24}
\end{array}\right) \rightarrow i\left(\begin{array}{ll}
r_{1} \sin t_{1} & r_{1} \cos t_{1} \\
r_{2} \cos t_{2} & r_{2} \sin t_{2}
\end{array}\right)
$$

and integrating out $\left(r_{1}, r_{2}, t_{2}\right)$, we obtain

$$
\mathcal{L}_{4}=\sum_{\sigma} \int \frac{d t}{4 i \sin t} \delta^{2 \mid 3}\left(\Lambda_{1}+i \sin t \Lambda_{3}+i \sigma \cos t \Lambda_{4}\right) \delta^{2 \mid 3}\left(\Lambda_{2}+i \cos t \Lambda_{3}-i \sigma \sin t \Lambda_{4}\right)
$$

in agreement with (2.24). Repeating the same analysis with pivot columns $\{1,4\}$, we find that $\mathcal{L}_{4}\left(\Lambda_{1}, \Lambda_{2}, \Lambda_{3}, \Lambda_{4}\right)$ reduces precisely to $A_{4}$ given by (2.26). Thus, the OG integral provides a geometric representation of the 4 -point vertex.

\subsection{Amalgamation and permutation}

An on-shell diagram for $k>2$ can be constructed by gluing two or more diagrams together. The corresponding $\mathrm{OG}_{k>2}$ is obtained by 'amalgamating' a collection of $\mathrm{OG}_{2}$ 's by integrating out the internal lines. The amalgamation proceeds as follows (see figure 3).

1. Put two on-shell diagrams together, preserving all external legs. The resulting $C$ matrix is a direct product of the two sub-matrices and thus lives in $\mathrm{OG}_{k+k^{\prime}}$.

2. Pick an external line from each diagram. Identify them by setting the one as $\Lambda_{I}$ and the other as $-i \Lambda_{I}$, and perform the integral (2.14). Since it reduces a number of external legs by 2 , the result must be an element of $\mathrm{OG}_{k+k^{\prime}-1}$.

Integrating out the internal line explicitly, we have

$$
\begin{aligned}
& \int d^{2 \mid 3} \Lambda_{I} \prod_{m=1}^{k} \delta^{2 \mid 3}\left(\sum_{i=1}^{2 k-1} c_{m i} \Lambda_{i}+c_{m I} \Lambda_{I}\right) \prod_{n=1}^{k^{\prime}} \delta^{2 \mid 3}\left(\sum_{j=1}^{2 k^{\prime}-1} c_{n j}^{\prime} \Lambda_{j}^{\prime}-i c_{n I}^{\prime} \Lambda_{I}\right) \\
& =c_{I 1} \prod_{m=2}^{k} \delta^{2 \mid 3}\left(\sum_{i=1}^{2 k-1}\left(c_{m i}-\frac{c_{1 i}}{c_{1 I}} c_{m I}\right) \Lambda_{i}\right) \prod_{n=1}^{k^{\prime}} \delta^{2 \mid 3}\left(\sum_{j}^{2 k^{\prime}-1} c_{n j}^{\prime} \Lambda_{j}^{\prime}+i \sum_{i=1}^{2 k-1} \frac{c_{1 i}}{c_{1 I}} c_{n I}^{\prime} \Lambda_{i}\right) .
\end{aligned}
$$



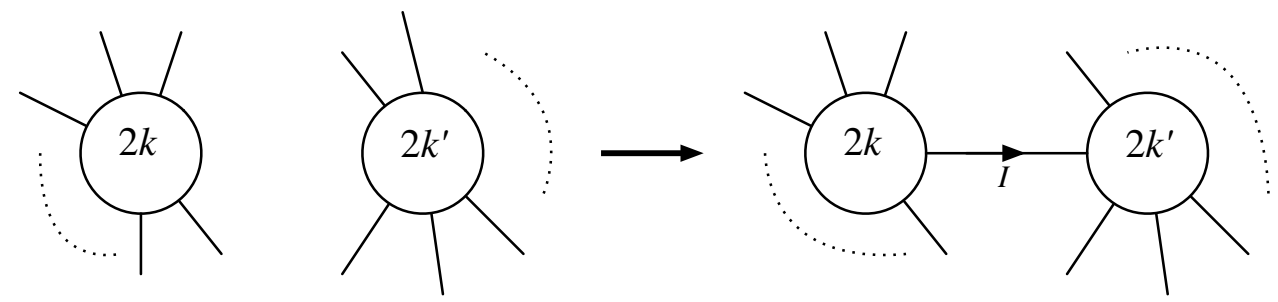

Figure 3. Gluing two on-shell diagrams.

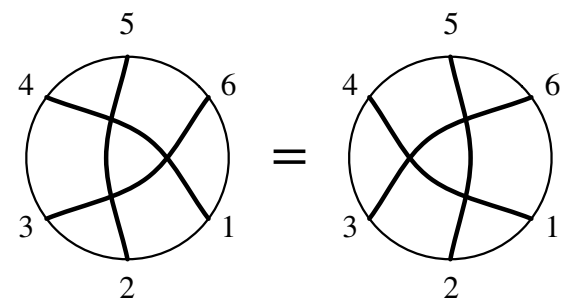

Figure 4. Yang-Baxter equivalence move.

The resulting matrix $\widetilde{C}$ is represented by

$$
\widetilde{C}_{\tilde{m} \tilde{\imath}}=\left(\begin{array}{c|c}
c_{m i}-\frac{c_{m I}}{c_{1 I}} c_{1 i} & 0 \\
\hline i \frac{c_{n I}^{\prime}}{c_{1 I}} c_{1 i} & c_{n j}^{\prime}
\end{array}\right)_{\tilde{m} \tilde{\imath}},
$$

where $\tilde{m}$ runs over $\left\{m=2 \cdots k, n=1 \cdots k^{\prime}\right\}$ and $\tilde{\imath}$ over $\left\{i=1 \cdots 2 k-1, j=1 \cdots 2 k^{\prime}-1\right\}$. As shown in [14], it is straightforward to verify that $\widetilde{C}$ respects the orthogonality condition $\widetilde{C} \cdot \tilde{C}^{T}=0$ if $C$ and $C^{\prime}$ satisfy the same condition,

$$
\sum_{i=1}^{2 k-1} c_{m i} c_{n i}+c_{m I} c_{n I}=0, \quad \sum_{j=1}^{2 k^{\prime}-1} c_{m j}^{\prime} c_{n j}^{\prime}+c_{m I}^{\prime} c_{n I}^{\prime}=0 .
$$

Equivalences move and reducible diagrams. Different on-shell diagrams corresponding to the same amplitude can be related to each other through a series of equivalence moves. The elementary move for ABJM amplitudes is the Yang-Baxter-like move depicted in figure 4. The lines 1 to 6 in the figure may be internal or external. In view of the BCFW bridging, the equivalence relation simply amounts to two different Euler angle decomposition of the same $\mathrm{SO}(3)$ rotation matrix.

As the name 'Yang-Baxter' suggests, the equivalence move leaves the permutation among external particles invariant. If we draw all possible diagrams with the same permutation content, we may encounter bubble diagrams such as those in figure 5. It was shown in [14] that the bubbles can be completely factorized from the rest of the on-shell diagram, leaving an integral with a $d \log$ measure. In the rest of this paper, we will work exclusively with bubble-free diagrams. 

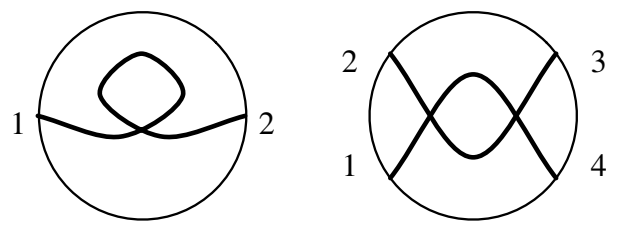

Figure 5. Diagrams containing bubbles.

\section{Reality and positivity of orthogonal Grassmannian}

The orthogonal Grassmannian $\mathrm{OG}_{k}$ is a subspace of $\mathrm{G}(k, 2 k)$ restricted by the orthogonality constraint. In this section, we shall examine the notion of reality and positivity for $\mathrm{OG}_{k}$. The real slice of $\mathrm{OG}_{k}$ is determined by the reality condition on external kinematic variables. For discussion on positivity, a particular reality condition, called the 'split signature' [14] condition, turns out to be the most convenient. Performing a 'Wick rotation' on the kinematic and the BCFW variables, we can rewrite the BCFW bridging rule with manifestly real and positive coordinates on $\mathrm{OG}_{k}$. Following [7, 14], we spell out the conversion rule which enables us to read off the postive $C$-matrix directly from an on-shell diagram without going through $\mathrm{BCFW}$ bridging one at a time.

\subsection{Complex OG}

Here we review the geometry of $\mathrm{OG}_{k}$ on which the integral (2.8) is defined [15]. Recall that the ordinary Grassmannian $\mathrm{G}(k, n)$ is the moduli space of $k$-planes in $n$ dimensions. In the standard matrix representation, $\mathrm{G}(k, n)$ is described by a $(k \times n)$ matrix $C$ with rank $k$ subject to the 'gauge symmetry' $C \sim g C$ with $g \in \mathrm{GL}(k) . \mathrm{OG}_{k}$ is a subspace of $\mathrm{G}(k, 2 k)$ subject to an 'orthogonality' constraint $\left(C \cdot C^{T}\right)_{m n}=C_{m i} C_{n i}=0(m=1, \ldots, k ; i=1, \ldots, 2 k)$. The constraint and the GL( $k)$ gauge symmetry determine the dimension of $\mathrm{OG}_{k}$ as

$$
\operatorname{dim}_{\mathbb{C}}\left[\mathrm{OG}_{k}\right]=2 k^{2}-k^{2}-\frac{k(k+1)}{2}=\frac{k(k-1)}{2} .
$$

It is also known that $\mathrm{OG}_{k}$ is isomorphic to the coset, $\mathrm{OG}_{k}=\mathrm{O}(2 k) / \mathrm{U}(k)$. Since $\mathrm{O}(2 k)$ contains two disjoint $\mathrm{SO}(2 k)$ components, $\mathrm{OG}_{k}$ is also decomposed into two disjoint subspaces. We will call them two 'branches' of $\mathrm{OG}_{k}$.

Let us discuss how the two branches are defined in terms of coordinates. Recall that the Plücker coordinates of $\mathrm{G}(k, n)$ are determinants of $(k \times k)$ submatrices of $C$, regarded as homogeneous coordinates of some projective space. The Plücker coordinates are subject to quadratic algebraic relations originating from linear dependencies among the columns of $C$. Coming back to $\mathrm{OG}_{k}$, let $I=\left\{i_{1}, i_{2}, \ldots, i_{k}\right\}$ be an ordered set of indices labeling $k$ distinct columns of $C$, and $M_{I}=\left(i_{1}, i_{2}, \cdots, i_{k}\right):=\operatorname{det}\left(C_{i_{1}}, C_{i_{2}}, \cdots, C_{i_{k}}\right)$ be the corresponding Plücker coordinate. As noted in [13], the orthogonality constraint $C \cdot C^{T}=0$ imposes linear relations among $M_{I}$ 's in addition to the quadratic relations for $\mathrm{G}(k, n)$. Define the complement of $I$ by $\bar{I}=\left\{\bar{\imath}_{1}, \bar{l}_{2}, \ldots, \bar{l}_{k}\right\}$ such that $\left\{i_{1}, \ldots, i_{k}, \bar{\imath}_{1}, \ldots, \bar{\imath}_{k}\right\}$ is an even permutation of $\{1,2, \ldots, 2 k\}$. The linear relation can be written as

$$
M_{I}=\sigma\left(i^{k}\right) M_{\bar{I}}
$$


The overall sign factor $\sigma= \pm 1$ defines the two branches of $\mathrm{OG}_{k}$. This linear relation implies a quadratic relation for consecutive minors on both branches [13],

$$
M_{i} M_{i+1}=M_{i+k} M_{i+1+k}(-1)^{k-1} .
$$

The orthogonality constraint can be expressed in terms of Plücker coordinates as

$$
\sum_{a}\left(i_{1}, \cdots, i_{k-1}, a\right)\left(j_{1}, \cdots, j_{k-1}, a\right)=0
$$

where $a$ runs over all columns of the $C$-matrix.

For $k=2$, we can solve all the relations explicitly. The two branches are defined by

$$
\mathrm{OG}_{k}^{ \pm}: \quad\left(M_{12}, M_{23}, M_{31}\right)=\mp\left(M_{34}, M_{14}, M_{24}\right) .
$$

Combining this with the Schouten identity,

$$
M_{12} M_{34}+M_{23} M_{14}+M_{31} M_{24}=0,
$$

and renaming the coordinates as $(X, Y, Z)=\left(M_{14}, M_{24}, M_{34}\right)$, we find that each of the two branches, $\mathrm{OG}_{2}^{ \pm}$, is described by an algebraic variety,

$$
\left\{(X, Y, Z) \in \mathbb{C P}^{2} \mid X^{2}+Y^{2}+Z^{2}=0\right\},
$$

which is topologically a $\mathbb{C P}^{1}$. We can compare this with the coset description,

$$
\mathrm{OG}_{2}^{+}=\mathrm{SO}(4) / \mathrm{U}(2) \simeq \mathrm{SU}(2) \times \mathrm{SU}(2) / \mathrm{U}(1) \times \mathrm{SU}(2) \simeq \mathrm{SU}(2) / \mathrm{U}(1)=\mathbb{C P}^{1} .
$$

Repeating the algebraic analysis for higher $k$ would be possible but quite cumbersome. For $k=3$, we can use the coset description to find

$$
\mathrm{OG}_{3}^{+}=\mathrm{SO}(6) / \mathrm{U}(3) \simeq \mathrm{SU}(4) / \mathrm{U}(3)=\mathbb{C P}^{3} .
$$

\subsection{Reality and positivity of OG}

Reality. In the spinor helicity formulation $p^{\alpha \beta}=\lambda^{\alpha} \lambda^{\beta}$ with real momentum, the spinor $\lambda^{\alpha}$ should be real or purely imaginary. Our convention is such that the spinor $\lambda^{\alpha}$ is real for outgoing particles and purely imaginary for incoming particles. If we want to work with strictly real momenta, we have to assign reality conditions on each of the external legs.

Momentum conservation forces all on-shell diagrams to have the same number of incoming and outgoing particles. First, the elementary 4-vertex (2.13) does not vanish only if two of the particles are incoming and the other two are outgoing. If all four particles are outgoing, the total momentum, $\lambda_{1} \lambda_{1}+\lambda_{2} \lambda_{2}+\lambda_{3} \lambda_{3}+\lambda_{4} \lambda_{4}$ is positive definite or negative definite, respectively, so cannot vanish. If particle $1,2,3$ are outgoing and 4 incoming, $\lambda_{1} \lambda_{1}+\lambda_{2} \lambda_{2}+\lambda_{3} \lambda_{3}$ generically has rank two while $\lambda_{4} \lambda_{4}$ has rank one, in conflict with momentum conservation. The other two unbalanced cases (4 incoming or 3 incoming +1 outgoing) can be treated similarly. Next, an internal line (2.14) always connects an incoming particle and an outgoing particle, hence the balance between incoming and outgoing particles continue to hold for arbitrary on-shell diagrams. 
The reality conditions for the kinematic variables $\lambda_{i}$ naturally translate into the reality conditions for the matrix $C$ representing a point on $\mathrm{OG}_{k}$. The linear delta function $\delta(C \cdot \Lambda)$ in (2.8) requires that

$$
\sum_{i=1}^{2 k} C_{m i} \lambda_{i}=0 .
$$

We may use the GL $(k)$ gauge symmetry to make the $k$ columns corresponding to incoming particles to form a $(k \times k)$ identity matrix, and denote the other $k$ columns by a non-trivial $(k \times k)$ matrix:

$$
\lambda_{i}+c_{i \bar{j}} \lambda_{\bar{j}}=0
$$

where the index $i$ runs over incoming particles and $\bar{j}$ over outgoing particles. Reality of $\lambda$ implies that $i c_{i \bar{j}}$ is a real matrix. The orthogonality constraint $C \cdot C^{T}=0$ then implies that $i c_{i \bar{j}}$ is an element of $\mathrm{O}(k, \mathbb{R})$. Thus, the reality condition for $\lambda_{i}$ defines a real slice of $\mathrm{OG}_{k}$. The two disconnected components of $\mathrm{O}(k, \mathbb{R})$ correspond to the two branches of $\mathrm{OG}_{k}$. For instance, the real slices of $k=3$ are two copies of $\mathrm{SO}(3)=\mathbb{R P}^{3}$.

We find it instructive to give a gauge invariant, geometric description of the reality conditions. We will focus on the simplest example for $k=2$. In the algebraic description (3.7), in a coordinate patch with $Z \neq 0$, we can consider four distinct reality conditions:

$$
\begin{array}{|c|cccc|}
\hline & (a) & (b) & (c) & (d) \\
\hline X / Z & i \mathbb{R} & \mathbb{R} & i \mathbb{R} & \mathbb{R} \\
Y / Z & \mathbb{R} & i \mathbb{R} & i \mathbb{R} & \mathbb{R} \\
\hline
\end{array}
$$

But, the condition (d) with $X^{2}+Y^{2}+Z^{2}=0$ yields an empty set in $\mathbb{C P}^{2}$. The three remaining real slices are identified with great circles, $S^{1}=\mathrm{SO}(2, \mathbb{R})$, embedded in $S^{2}=\mathbb{C P}^{1}$ as depicted in figure 6 . The intersections among different real slices are denoted as

$$
A_{ \pm}: X=0, Y / Z= \pm i, \quad B_{ \pm}: Y=0, Z / X= \pm i, C_{ \pm}: Z=0, X / Y= \pm i .
$$

We should emphasize that the reality condition is a gauge invariant notion. Although it is sometimes useful to align the gauge choice with the reality condition as in (3.11), other gauge choices might be more convenient for certain purposes. In section 4 , we will use gauge choices different from (3.11).

Positivity. As pointed out in [14], the 'split signature' reality condition, in which all odd-labelled particles are incoming and all even-labelled ones outgoing, deserves a special attention. It is the only reality condition that respects the cyclic symmetry of $A_{2 k}(2.7)$. Moreover, the split signature allows for a simple notion of 'positivity'. Following [14], we do a 'Wick rotation' on the $C$-matrix and $\lambda_{2 i-1}$ simultaneously such that all elements of $C$ and all $\lambda_{i}$ are real, while the orthogonality constraint takes the form,

$$
C \cdot \eta \cdot C^{T}=0, \quad \eta_{i j}=(-1)^{i} \delta_{i j}=\operatorname{diag}(-,+, \cdots,-,+) .
$$

In this convention, the positivity defined in [14] asserts that all ordered minors of $C$ are non-negative. ${ }^{2}$ This particular definition of positivity picks out one of the two branches of

\footnotetext{
${ }^{2} \mathrm{~A}$ similar notion for $\mathrm{G}(k, n)$ is called 'totally non-negative' in mathematics literature [16-18]. For brevity, we will write 'positive' in place of 'totally non-negative'.
} 


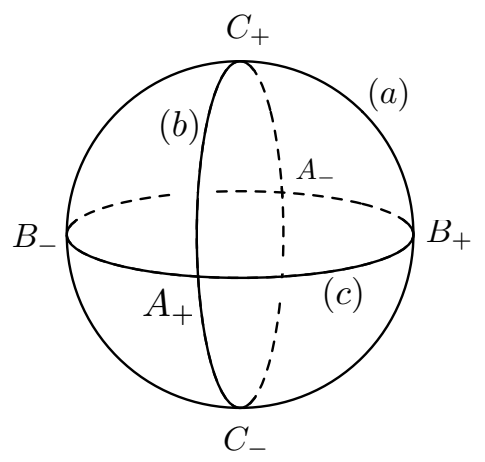

Figure 6. Real slices of a $\mathrm{OG}_{2+}$.

$\mathrm{OG}_{k}$ at each $k$. For instance, we have

$$
(1,2, \ldots, k)=(k+1, k+2, \ldots, 2 k) \text { and }(1,3, \ldots, 2 k-1)=(2,4, \ldots, 2 k) .
$$

It was shown in [14] that the definition of positivity is compatible with all essential properties of OG such as (3.3) and (3.4).

These results strongly suggest that the positive orthogonal Grassmannian (POG) has the same dimension as the real slice of OG. One of the main goals of this paper is to introduce a complete set of coordinate patches for POG for all $k$. We give a simplest example $(k=2)$ here for illustration and discuss the general construction in section 4 . Let us choose a gauge such that

$$
C=\left(\begin{array}{llll}
1 & 0 & c_{13} & c_{14} \\
0 & 1 & c_{23} & c_{24}
\end{array}\right) .
$$

In the split signature, $\eta=\operatorname{diag}(-,+,-,+)$, the orthogonality constraint $C \cdot \eta \cdot C^{T}=0$ gives

$$
-1-c_{13}^{2}+c_{14}^{2}=0, \quad 1-c_{23}^{2}+c_{24}^{2}=0, \quad-c_{13} c_{23}+c_{14} c_{24}=0 .
$$

Positivity requires that

$$
c_{13}, c_{14} \leq 0, \quad c_{13}, c_{14} \geq 0 .
$$

The complete solution to this problem is

$$
\left(\begin{array}{ll}
c_{13} & c_{14} \\
c_{23} & c_{24}
\end{array}\right)=\left(\begin{array}{cc}
-\sinh t & -\cosh t \\
\cosh t & \sinh t
\end{array}\right), \quad t \geq 0 .
$$

As $t$ approaches $\infty$, we can take a gauge transformation to find

$$
C=\left(\begin{array}{cc}
1 & -\sinh t \\
0 & \cosh t
\end{array}\right)\left(\begin{array}{cccc}
1 & \tanh t & 0 & -\operatorname{sech} t \\
0 & \operatorname{sech} t & 1 & \tanh t
\end{array}\right) \rightarrow\left(\begin{array}{llll}
1 & 1 & 0 & 0 \\
0 & 0 & 1 & 1
\end{array}\right)
$$

Including the 'point' at $t=+\infty$, the full geometry of $\mathrm{POG}_{2}$ is an interval with two endpoints included. In figure 6 , the $\mathrm{POG}_{2}$ is identified with the interval $\overline{A_{-} C_{+}}$. 


\subsection{Conversion rule}

Recall that the elementary vertex, $A_{4}$, is resolved in two ways, (2.24) and (2.26), according to the choice of pivot columns for $\mathrm{OG}_{2}$. The first one (2.24) contains a delta-function,

$$
\delta^{2 \mid 3}\left(\Lambda_{1}+i \sin t \Lambda_{3}+i \sigma \cos t \Lambda_{4}\right) \delta^{2 \mid 3}\left(\Lambda_{2}+i \cos t \Lambda_{3}-i \sigma \sin t \Lambda_{4}\right) .
$$

We perform a Wick rotation on the odd-labelled particles as $\Lambda_{2 i-1} \rightarrow-i \Lambda_{2 i-1}$ and on the BCFW variables as $t \rightarrow i t$. After the Wick rotation, up to an overall phase, the delta-function becomes

$$
\delta^{2 \mid 3}\left(\Lambda_{1}-\sinh t \Lambda_{3}-\sigma \cosh t \Lambda_{4}\right) \delta^{2 \mid 3}\left(\Lambda_{2}+\cosh t \Lambda_{3}+\sigma \sinh t \Lambda_{4}\right) .
$$

Similarly, in the other case (2.26), the Wick rotation gives

$$
\begin{aligned}
& \delta^{2 \mid 3}\left(\Lambda_{1}+i \sigma \cos t \Lambda_{2}-i \sin t \Lambda_{3}\right) \delta^{2 \mid 3}\left(\Lambda_{4}-i \sigma \sin t \Lambda_{2}-i \cos t \Lambda_{3}\right) \\
\rightarrow & \delta^{2 \mid 3}\left(\Lambda_{1}-\sigma \cosh t \Lambda_{2}+\sinh t \Lambda_{3}\right) \delta^{2 \mid 3}\left(\Lambda_{4}+\sigma \sinh t \Lambda_{2}-\cosh t \Lambda_{3}\right) .
\end{aligned}
$$

We apply the same Wick rotation to the internal line (2.14) as well,

$$
\delta\left(i \Lambda_{1}+\Lambda_{2}\right) \quad \rightarrow \quad \delta\left(\Lambda_{1}+\Lambda_{2}\right)
$$

where $\Lambda_{1}$ and $\Lambda_{2}$ represent odd and even multiplets, respectively.

As explained in $[7,14]$, we can collect the linear relations imposed by the delta-functions and read off the components of the $C$-matrix without going through BCFW bridging and amalgamation one at a time. To find the component $c_{i \bar{j}}$ in the gauge-fixed form, $\lambda_{i}+c_{i \bar{j}} \lambda_{\bar{j}}=$ 0 , we trace all possible paths $p$ from the source $i$ to the sink $\bar{j}$ in the corresponding on-shell diagram. Each internal line (3.24) contributes a factor of $(-1)$, since $\delta\left(\lambda_{1}+\lambda_{2}\right)$ implies $\lambda_{1}=-\lambda_{2}$. At each vertex, we pick up $\left(-f_{v}\right)$, where $f_{v}$ is one of the matrix elements of the $(2 \times 2)$ matrix defined at the vertex, $\lambda_{i}+c_{i \bar{j}}^{(v)} \lambda_{\bar{j}}=0$, chosen by how the path traverses the vertex. ${ }^{3}$ The vertex factors in the two cases of BCFW bridging considered above are summarized in figure 7. The factors in figure 7(a) are read off from (3.22) and those in figure 7 (b) from (3.23). Combining the contributions from internal lines and vertices, we arrive at a simple conversion rule for $c_{i \bar{j}}$ :

$$
c_{i \bar{j}}=-\sum_{p \in\{i \rightarrow \bar{j}\}}\left(\prod_{l \in p}(-1) \cdot \prod_{v \in p}\left(-f_{v}\right)\right)=\sum_{p \in\{i \rightarrow \bar{j}\}}\left(\prod_{v \in p} f_{v}\right) .
$$

The matrix elements are manifestly real. The factors of $(-1)$ have cancelled out completely, since a path always traverse $n_{p}$ internal lines and $n_{p}+1$ vertices. We will study how positivity restricts the matrix elements in the next section.

\footnotetext{
${ }^{3}$ The minus sign in $\left(-f_{v}\right)$ arises because we view the relation $\lambda_{i}+c_{i \bar{j}}^{(v)} \lambda_{\bar{j}}=0$ as $\lambda_{i}=-c_{i \bar{j}}^{(v)} \lambda_{\bar{j}}$. The overall minus sign right after the first equality sign in (3.25) is inserted for the same reason.
} 


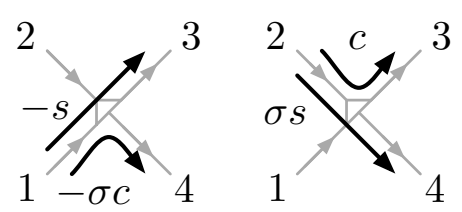

(a)

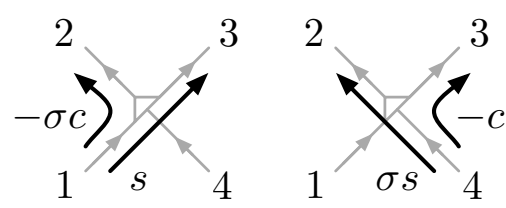

(b)

Figure 7. Vertex factors $f_{v}$ in the canonical gauge to be used in the conversion rule.

\section{Positroid stratification}

The 'positroid stratification' of $\mathrm{G}_{+}(k, n)$ was developed in $[16,17]$ and brought to physics in [7]. It relates the combinatorics of on-shell diagrams to the geometry of $\mathrm{G}_{+}(k, n)$. At an intermediate step, a notation similar to Young tableaux plays a crucial role, which encodes the linear dependency among the columns of the matrix representative of $\mathrm{G}_{+}(k, n)$. In this section, we shall develop a similar story for the positive stratification of OG. Some partial results in this direction were obtained in [14].

As a first step, we introduce an auxilary aid called 'OG tableau' which encodes the combinatorics of on-shell diagrams. It naturally provides a set of canonical gauge choices for the $C$-matrix such that the restriction imposed by positivity takes a simple form. With the help of the OG tabeaux, we construct the 'canonical' coordinate system, which exhibits positivity by construction for each cell of $\mathrm{POG}_{k}$ for all $k$. We verify that the canonical coordiates obtained from the positive stratification agrees with the ones given by the conversion rule derived in section 3 .

In the last subsection, we turn to the mathematics of $\mathrm{POG}_{k}$. It is known that the positive Grassmannian $\mathrm{G}_{+}(k, n)$ forms a combinatorial polytope called 'Eulerian poset' for each $(k, n)$ [19]. The graded counting of OG tableaux suggests that $\mathrm{POG}_{k}$ may also define an Eulerian poset for each $k$. Geometrically, it seems plausible that $\mathrm{POG}_{k}$ has a topology of a ball. We verify this conjecture for $k=2,3$. Finally, we give a preliminary discussion on the boundary operation on $\mathrm{POG}_{k}$. We expect that a more complete study of the boundary operation will help us better understand the topology and geometry of POG.

\subsection{OG tableaux}

As we discussed earlier, on-shell diagrams are determined by a splitting of $\{1,2, \ldots, 2 k\}$ into $k$ pairs of integers, $\left\{\left(a_{1} b_{1}\right), \cdots,\left(a_{k} b_{k}\right)\right\}$. By convention, we set $a_{m}<b_{m}$ for all $m$. Barring bubbles and modulo Yang-Baxter equivalence moves, the diagrams are in oneto-one correspondence with the pairings. The total number of inequivalent diagrams are $(2 k) ! /\left(2^{k} k !\right)=1,3,15,105, \cdots$. Some subclasses of diagrams are easy to enumerate. For a given $k$, there is a unique 'top' diagram with the maximal number, $k(k-1) / 2$, of vertices (see figure 8). All external legs in a top diagram are paired diagonally.

At the opposite extreme, there are 'bottom' diagrams with no vertex (see figure 9). The counting of non-intersecting diagrams connecting $2 k$ cyclically ordered points is an 

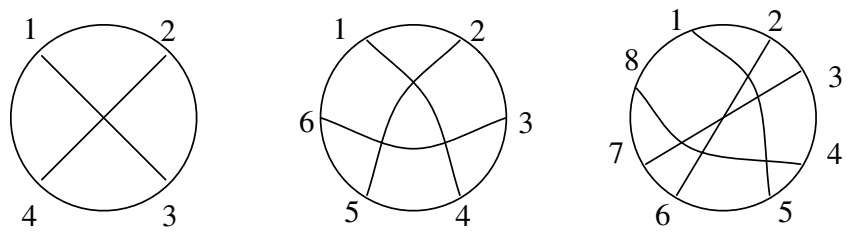

Figure 8. Top-cell diagrams for $k=2,3,4$.
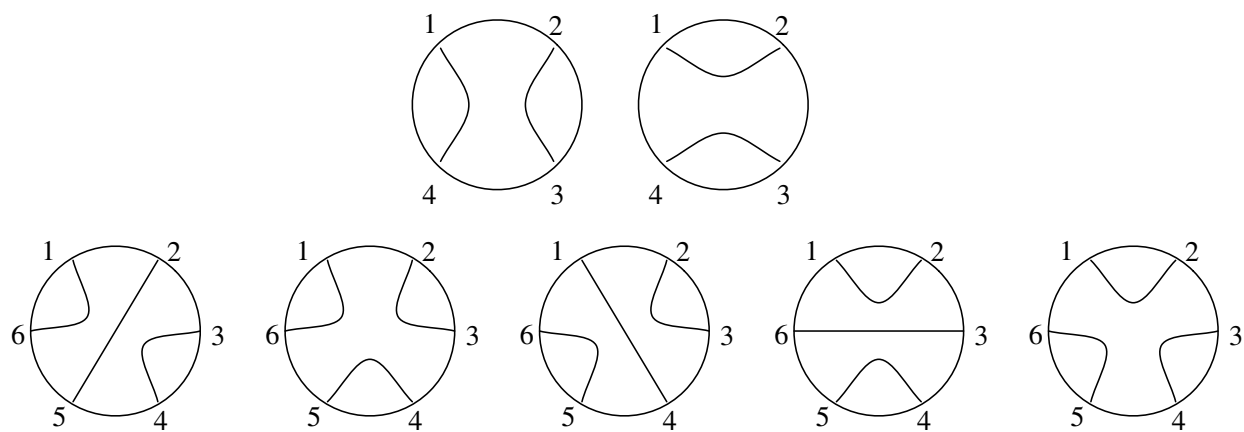

Figure 9. Bottom-cell diagrams for $k=2$ (upstairs) and $k=3$ (downstairs).

elementary problem in combinatorics. The answer is the $k^{\prime}$ th Catalan number $\mathrm{C}_{k}[35]$,

$$
\mathrm{C}_{k}=\frac{1}{k+1}\left(\begin{array}{c}
2 k \\
k
\end{array}\right)=\frac{(2 k) !}{(k+1) ! k !}=1,2,5,14,42, \cdots .
$$

To enumerate the diagrams with intermediate number of vertices, and relate them to subspaces of $\mathrm{OG}_{k}$, we introduce a new notation called 'OG tableaux'. There are two related versions of the tableaux: 'unfolded' and 'folded'.

Unfolded tableaux. Figure 10 illustrates how to map an on-shell diagram to a tableau with an example. We first prepare the off-diagonal upper-left half of a $(2 k \times 2 k)$ chessboard. The diagonal boxes of the chessboard are numbered from 1 to $2 k$, with 1 placed at the lowerleft corner and $2 k$ at the upper-right corner. The empty tableau contains $2 k(2 k-1) / 2$ boxes, in one-to-one correspondence with a pair chosen from $\{1, \ldots, 2 k\}$. For each pair $\left(a_{m} b_{m}\right) \in\left\{\left(a_{1} b_{1}\right), \cdots,\left(a_{k} b_{k}\right)\right\}$, we put a 'hook' on the corresponding box. If we extend the right/lower arm of the hook horizontally/vertically toward the diagonal, we recover precisely a copy of the on-shell diagram. So far, the only novelty of the tableau notation is that it defines a canonical way to fix the Yang-Baxter ambiguity.

Folded tableaux. It is possible to 'fold' the unfolded tableaux without reducing its information content. We begin with examining each of the $(k-1)$ columns and $(k-1)$ rows of the unfolded tableaux. If a column/row contains no hook, all the boxes in the column/row are removed. The surviving boxes can be moved in horizontal or vertical directions and fit into a $(k \times k)$ chessboard. See figure 11 for an illustration.

The content of an on-shell diagram is preserved through the folding procedure, so the map between on-shell diagrams and folded tableaux is still bijective. The labels for source 


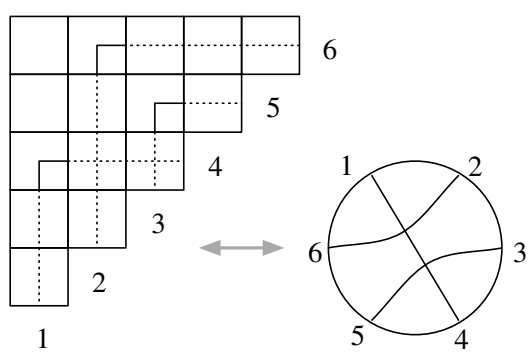

(a)

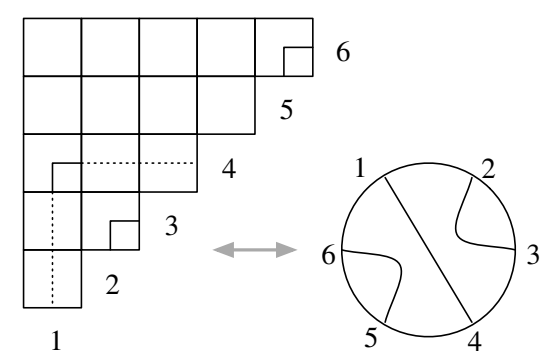

(b)

Figure 10. Examples of unfolded $\mathrm{OG}_{3}$ tableaux.

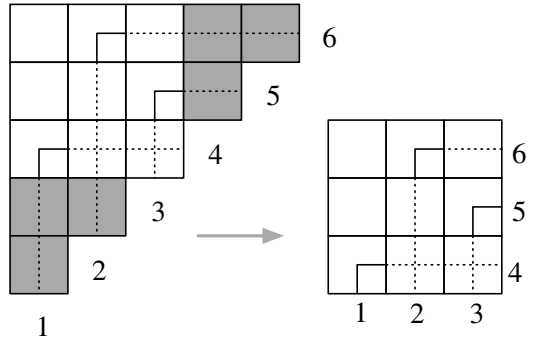

(a)

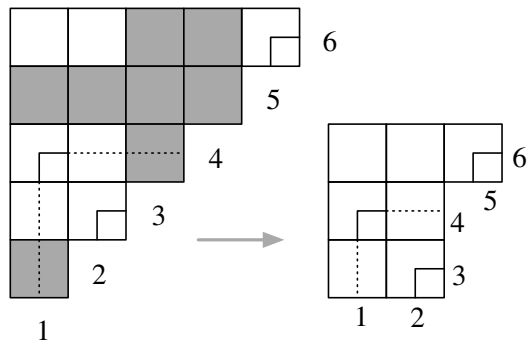

(b)

Figure 11. Folding the $\mathrm{OG}_{3}$ tableaux.

legs $\left\{a_{i}\right\}$ are attached to the boundaries at the bottom edges of the folded tableau, while those for sink legs $\left\{b_{i}\right\}$ are attached to the right edges. Thus, on-shell diagrams with the same set of sink/source legs share the same configuration of boxes for the folded tableaux, but are distinguished by the placement of hooks.

One of the fundamental feature of an on-shell diagram is its number of vertices ('level'). We can classify the on-shell diagrams according to the type of the corresponding (folded) tableau and the level. The full classification for $k=2,3$ is given in figure 12 .

We introduced the unfolded tableaux first and switched to the folded tableaux for a pedagogical reason. But, it is no more difficult to work directly with the folded tableaux. We shall construct the $\mathrm{OG}_{k}$ tableaux as follows (see figure 13).

1. Draw a $(k \times k)$ chessboard.

2. Remove some boxes among the $k(k-1) / 2$ boxes in the lower-right off-diagonal half, such that the remaining boxes form a Young tableau of at least $k(k+1) / 2$ boxes.

3. Assign 1 to $2 k$ to the bottom and right edges of the stack of boxes in order.

4. Mark a box with a hook for each column, under the restriction that each row should contain one and only one hook.

Each marked tableau gives an on-shell diagram. Its level can be determined as follows.

1. Count how many boxes were removed from the $(k \times k)$ chessboard. 
(3)

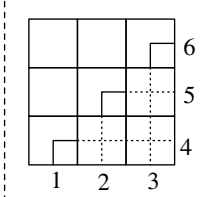

(2)

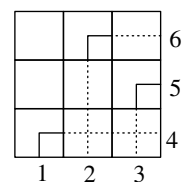

(1)

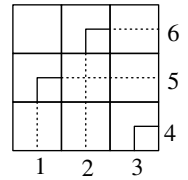

(0)

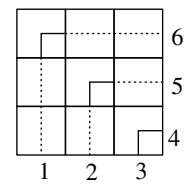

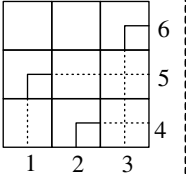
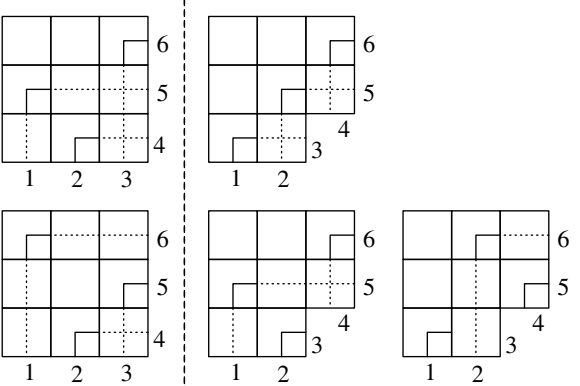

$\{1,2,3\}$

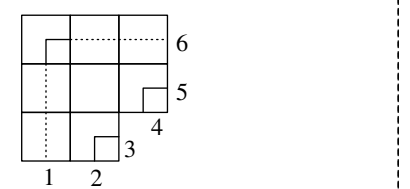

$\{1,2,4\}$

Figure 12. All diagrams for $k=2,3$ classified by folded tableaux and levels.
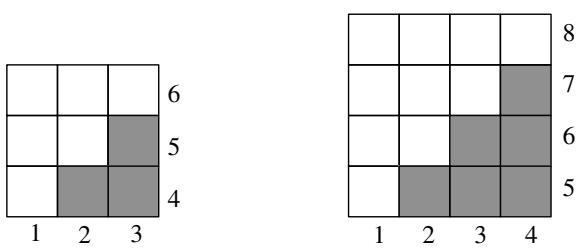

Figure 13. Shaded boxes are removable.

2. Compute the number of row permutations required to arrange marked boxes diagonally from the bottom left corner to the top right.

3. Add up the two numbers, then subtract it from $k(k-1) / 2$.

As a special case, a top diagram requires no removal of boxes or rearrangement of marked boxes. So, the prescription above gives the expected level $k(k-1) / 2$. The readers are invited to test the prescription against less trivial examples in figure 12.

\subsection{Canonically positive coordinates}

Positroid stratification relates the combinatorics of on-shell diagrams and OG tableaux to the geometry of $\mathrm{POG}_{k}$. Each tableau is mapped to a subspace of $\mathrm{POG}_{k}$. Importing the terminology from the positroid stratification of $\mathrm{G}(k, n)[16,17]$, we will call the subspaces 'cells' of $\mathrm{POG}_{k}$. The number of vertices of an on-shell diagram (level of its tableau) equals the dimension of the cell. In this subsection, we will introduce a canonical coordinate system to the cells. The cells sharing the same unmarked tableau (see figure 12) will share a common coordinate patch. 


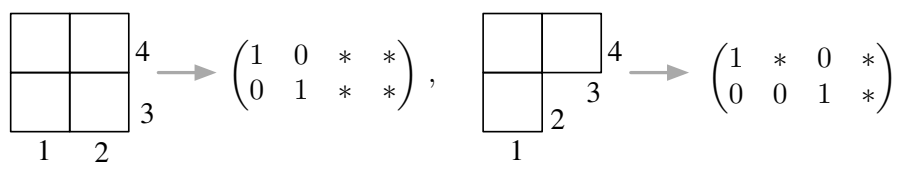

Figure 14. Source legs of tableaux translate into pivot columns of $C$-matrices.

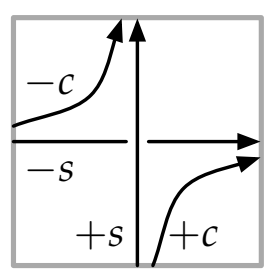

(b) BCFW bridge

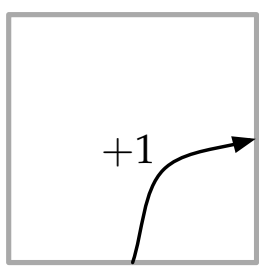

(a) hook $c=\cosh t$

$s=\sinh t$.

Figure 15. Rules for reading off matrix elements from a tableau.

From tableaux to matrices. The (folded) OG tableaux reveals a decomposition of $\mathrm{OG}_{k}$ similar to the standard Schubert decomposition of $\mathrm{G}(k, n)$. The source legs translate into 'pivot' columns. Let $\left\{p_{m}\right\}(m=1, \ldots, k)$ be a monotonically increasing labels for the source legs. We put $C$ into a row-echelon form by setting $C_{m, p_{m}}=1, C_{m, i<p_{m}}=0$ and $C_{n \neq m, p_{m}}=0$. An example is given in figure 14 .

Note that using the $\mathrm{GL}(k)$ gauge symmetry, we can write every element of $\mathrm{OG}_{k}$ in the row-echelon form. The whole $\mathrm{OG}_{k}$ can be written as a disjoint union, $\mathrm{OG}_{k}=\bigsqcup_{\lambda} \Omega_{\lambda}$, where $\lambda$ runs over unmarked tableaux. One can interpret $\Omega_{\lambda}$ as disjoint coordinate patches, covering the whole $\mathrm{OG}_{k}$, then an on-shell diagram always belongs to a particular $\lambda$.

Unlike the Schubert decomposition of $\mathrm{G}(k, n)$, in the current setup, the orthogonality constraint restricts the allowed set of pivot columns. Since the folded tableaux descend from the unfolded tableaux which in turn are copied from allowed on-shell diagrams, the folded tableaux naturally capture the allowed sets of pivot columns.

The matrix elements of non-pivot columns are determined by adopting the conversion rule introduced in section 3.3 and modifying it slightly to fit into the tableaux notation. Two modifications are needed. First, since the definition of positivity requires a specific branch (3.15), we are forced to select the orientation-preserving BCFW bridges: $\sigma=+1$ in figure $7(\mathrm{a})$ and $\sigma=-1$ in figure $7(\mathrm{~b})$. Second, the labels for external legs are ordered clockwise in on-shell diagrams but counter-clockwise on OG tableaux. Taking these factors into account, we arrive at a remarkably simple final rule, depicted in figure 15. Note that the two types of bridges in figure 7 has been unified into a single one in figure 15 . An example of the application of the conversion rule is given in figure 16 .

At this point, it is not clear how the positivity of Plücker coordinate is related to the positivity of BCFW variables appearing in the conversion rule. To reveal the connection, we will turn to an equivalent, and often more convenient, way to determine the matrix elements. We will begin with bottom cells whose matrix elements are completely fixed by positivty. We will then successively turn on BCFW bridges by multiplying the $C$-matrix from the right by an $\mathrm{SO}(k, k)$ rotation matrix. 


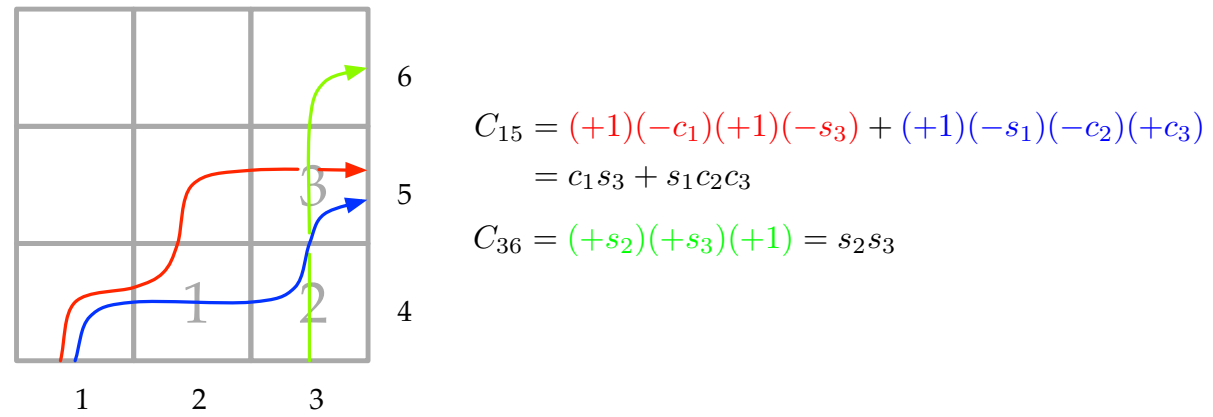

Figure 16. Reading off matrix elements from a tableau: an example.

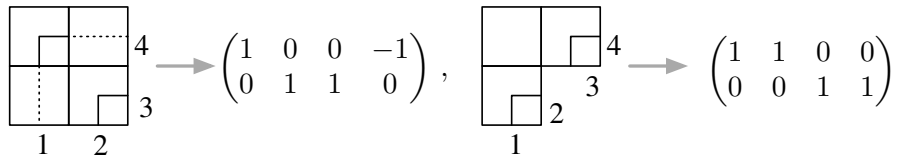

Figure 17. The $C$-matrices for 0 -cycles at $k=2$.

Bottom cells. Each unmarked tableau hosts a unique bottom cell (see figure 12). Given an unmarked tableau with pivot columns $\left\{p_{m}\right\}$, let $\left\{q_{m}\right\}$ be the labels for the 'sink' columns. By construction, $C_{m, p_{m}}=1$ and $C_{m, i}=0$ for $i \neq p_{m}, q_{m}$. The orthogonality constraint requires that $C_{m, q_{m}}= \pm 1$. Positivity determines the sign of $C_{m, q_{m}}$ uniquely. For bottom cells, a minor is non-vanishing if and only if it contains either a pivot column $p_{m}$ or its sink column $q_{m}$ but not both or neither. We start with $\left(p_{1}, p_{2}, \cdots, p_{k}\right)=1$, which is positive by construction. Suppose we replace a pivot column $p_{m}$ with its sink column $q_{m}$. If the two columns are adjacent $\left(q_{m}=p_{m}+1\right)$, the ordering of the columns in the minor will be preserved, and the minor will remain positive if and only if $C_{m, q_{m}}=+1$. Suppose now $p_{m}$ and $q_{m}$ are not adjacent. Since we are dealing with bottom diagrams with no intersection among lines, the interval between $p_{m}$ and $q_{m}$ may contain a pair $\left(p_{n}, q_{n}\right)$ for some $n$, but not $p_{n}$ or $q_{n}$ separately. We can recover the ordering of the minor by shifting the column $q_{m}$ to the right by $\left(q_{m}-p_{m}-1\right) / 2$ steps. The resulting ordered minor will become positive if and only if we set

$$
C_{m, q_{m}}=(-1)^{\left(q_{m}-p_{m}-1\right) / 2} .
$$

See figure 17 for the explicit form of $C$ matrices for the bottom diagrams at $k=2$.

BCFW rotation. Having specified the bottom cells, we can start turning on the BCFW bridges. The $\mathrm{BCFW}$ bridges act on the $C$-matrix by a right multiplication of an $\mathrm{SO}(k, k)$ 'rotation'. The rotations act only on the sink columns and leave the pivot columns intact.

When there are two or more BCFW bridges, the order of the rotation matrices can be determined as follows. Let us define the 'floor' of a pivot column as their vertical distance from the bottom of the OG tableau. For example, in figure 11(b), pivot columns 1 and 2 reside on the 0th floor, while column 5 resides on the 2 nd floor.

The BCFW bridging begins with those pivot columns on the 0th floor. We bridge the two left-most pivot columns, say 1 and 2 , in the sense that the rotation matrix acts on the 


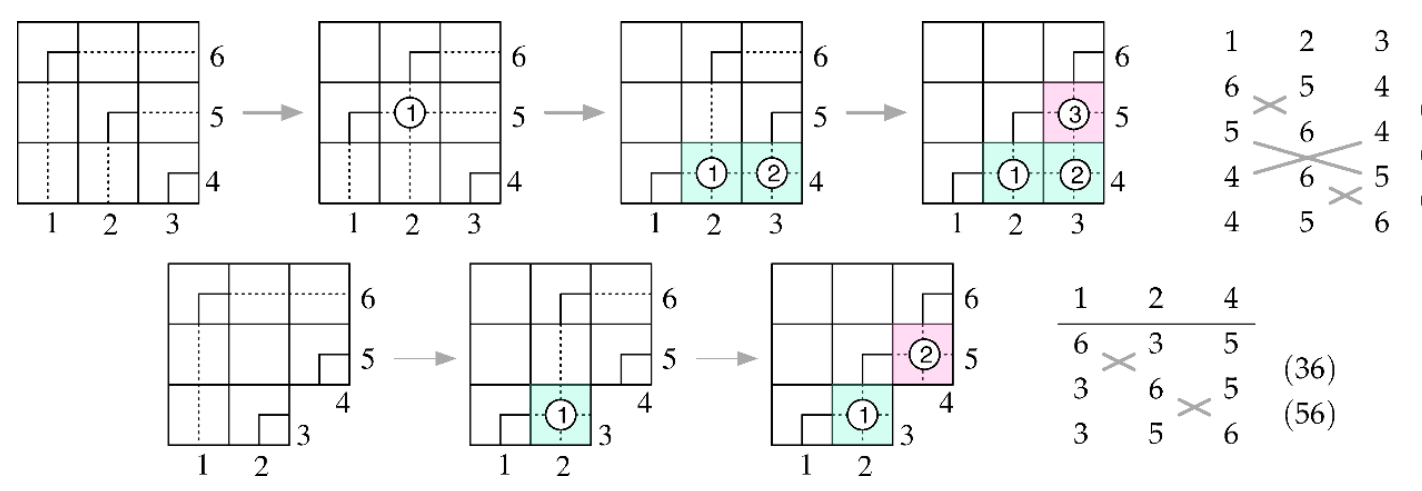

Figure 18. BCFW bridging of the top cell (above) and level 2 cell (below) for $k=3$.
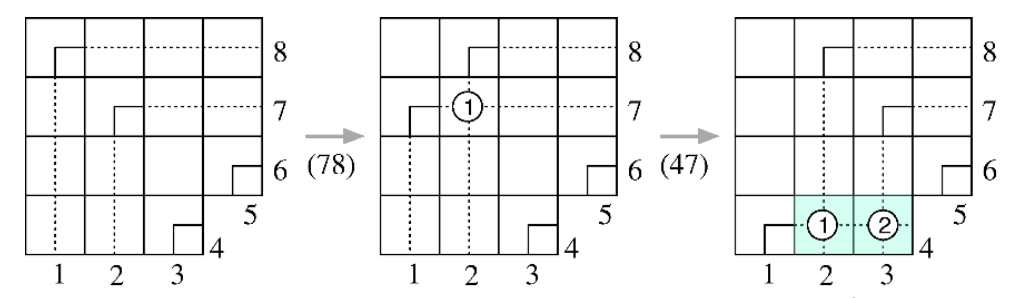

(78)
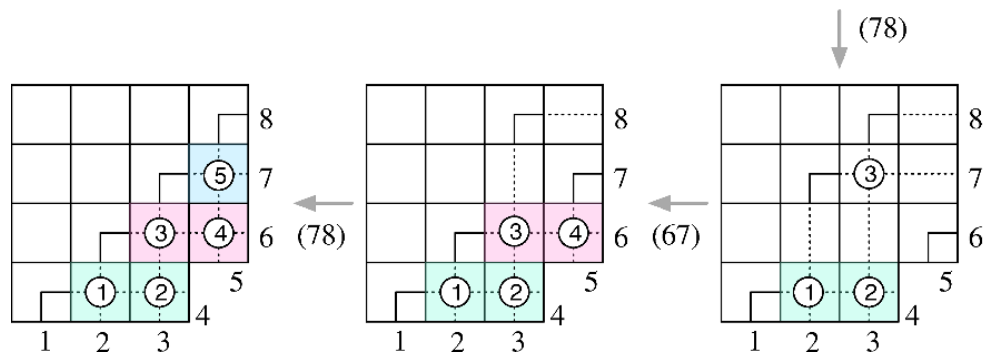

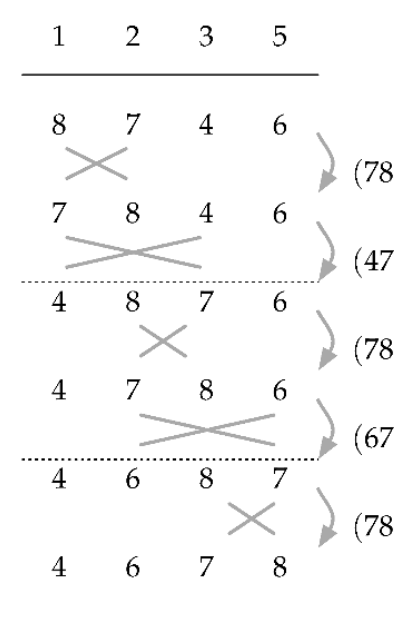

Figure 19. BCFW bridging of the level-5 cell for $k=4$.

corresponding sink columns. The hook above pivot 1 is lowered from its original location. If there are more pivot columns on the 0th floor, say column 3 , then we bridge pivots 1 and 3 . We continue the process until the hook above pivot 1 comes down to the 0th floor. Finally, we decouple column 1 and elevate other columns on the 0th floor to the 1st floor.

We proceed in the same way on the 1st floor including those elevated from the 0th floor and those born on the 1st floor. The second leftmost column from the 0th floor, if exists, becomes the leftmost column of the 1st floor. The final result can be summarized in a simple way. Given an empty OG tableau, to go from level 0 to the highest level, we perform the BCFW bridging such that the order or rotation is read off from left to right on the 0th floor, and then from left to right on the 1st floor, and so on. Two examples are given in figure 18 and figure 19.

Positivity. A rotation matrix $R$ for swapping a pair of sink columns $c_{i}$ and $c_{j}(i<j)$ is the tensor product of the non-trivial $2 \times 2$ block,

$$
R_{i i}=R_{j j}=\cosh t, \quad R_{i j}=R_{j i}=(-1)^{(j-i-1) / 2} \sinh t,
$$


and a $(2 k-2) \times(2 k-2)$ identity matrix. The sign factor in (4.3), which resembles (4.2), has been inserted to preserve positivity for $t \geq 0$. The rotation swaps the sink columns $c_{i}$ and $c_{j}$. It leads to mixing of the Plücker coordinates $(\cdots i \cdots)$ and $(\cdots j \cdots)$,

$$
\begin{aligned}
& (\cdots i \cdots) \rightarrow R_{i i}(\cdots i \cdots)+R_{i j}(\cdots j \cdots) \\
& (\cdots j \cdots) \rightarrow R_{j i}(\cdots i \cdots)+R_{j j}(\cdots j \cdots)
\end{aligned}
$$

When $c_{i}$ and $c_{j}$ are adjacent $(j=i+1)$, the two minors $(\cdots i \cdots)$ and $(\cdots j \cdots)$ share a common ordering. Thus, to ensure positivity of minors after the rotation, we should require that $R_{i j} \geq 0$. When $c_{i}$ and $c_{j}$ are not adjacent, the ordering of $(\cdots i \cdots)$ and $(\cdots j \cdots)$ differ precisely by the sign factor $(-1)^{(j-i-1) / 2}$, such that the rotation (4.3) with the sign factor preserves positivity. Finally, we note that

$$
\begin{aligned}
(\cdots i \cdots j \cdots) \rightarrow & \cosh ^{2} t(\cdots i \cdots j \cdots)+\sinh ^{2} t(\cdots j \cdots i \cdots) \\
& =\left(\cosh ^{2} t-\sinh ^{2} t\right)(\cdots i \cdots j \cdots)=(\cdots i \cdots j \cdots) .
\end{aligned}
$$

We give an explicit form of the $C$-matrix for the two examples shown in figure 18. Since the pivot columns form a $(k \times k)$ identity matrix by construction, it suffices to present the sink columns. For the $k=3$, level 2 cell with pivot columns $\{1,2,4\}$, we have

$$
\left(C_{3}, C_{5}, C_{6}\right)=\left(\begin{array}{lll}
0 & 0 & 1 \\
1 & 0 & 0 \\
0 & 1 & 0
\end{array}\right)\left(\begin{array}{ccc}
c_{1} & 0 & -s_{1} \\
0 & 1 & 0 \\
-s_{1} & 0 & c_{1}
\end{array}\right)\left(\begin{array}{ccc}
1 & 0 & 0 \\
0 & c_{2} & s_{2} \\
0 & s_{2} & c_{2}
\end{array}\right)=\left(\begin{array}{ccc}
-s_{1} & c_{1} s_{2} & c_{1} c_{2} \\
c_{1} & -s_{1} s_{2} & -s_{1} c_{2} \\
0 & c_{2} & s_{2}
\end{array}\right)
$$

where we used the notations $c_{i}=\cosh t_{i}, s_{i}=\sinh t_{i}$. For the $k=3$ top-cell with pivot columns $\{1,2,3\}$, we have

$$
\begin{aligned}
\left(C_{4}, C_{5}, C_{6}\right) & =\left(\begin{array}{ccc}
0 & 0 & 1 \\
0 & -1 & 0 \\
1 & 0 & 0
\end{array}\right)\left(\begin{array}{ccc}
1 & 0 & 0 \\
0 & c_{1} & s_{1} \\
0 & s_{1} & c_{1}
\end{array}\right)\left(\begin{array}{ccc}
c_{2} & s_{2} & 0 \\
s_{2} & c_{2} & 0 \\
0 & 0 & 1
\end{array}\right)\left(\begin{array}{ccc}
1 & 0 & 0 \\
0 & c_{3} & s_{3} \\
0 & s_{3} & c_{3}
\end{array}\right) \\
& =\left(\begin{array}{ccc}
s_{1} s_{2} & s_{1} c_{2} c_{3}+c_{1} s_{3} & c_{1} c_{3}+s_{1} c_{2} s_{3} \\
-c_{1} s_{2} & -c_{1} c_{2} c_{3}-s_{1} s_{3} & -s_{1} c_{3}-c_{1} c_{2} s_{3} \\
c_{2} & s_{2} c_{3} & s_{2} s_{3}
\end{array}\right) .
\end{aligned}
$$

It is straightforward to verify that all ordered minors of the corresponding $C$-matrices are manifestly non-negative, provided that $t_{i} \geq 0$.

We have described two ways to construct the $C$-matrix. One is to use the conversion rule summarized in figure 15 and the other is to perform a sequence of BCFW rotations. The latter exhibits manifest positivity, while the former reveals the connection to on-shell diagrams more clearly. As an astute reader may have expected, with hindsight, we have adjusted the variables in the two approaches such that the they agree without any change of variables. Although we have not been able to find a general proof for this agreement, we have verified it in all examples up to $k=5$ and expect that it will hold for all $k$. 
Integration measure. We note in passing that the integration measure is factorized into a $d \log$ form in a way similar to that of [7]. Taking the measures from the elementary BCFW vertices (2.24), (2.26) and taking account of Wick rotation, we see that the integration measure for the full Grassmannian integral can be written as

$$
\int \prod_{i} \frac{d t_{i}}{\sinh t_{i}}=\int \prod_{i} \frac{d z_{i}}{z_{i}}=\int \prod_{i} d \log z_{i} \quad\left(z_{i}:=\tanh \frac{t_{i}}{2}\right) .
$$

\subsection{Polytope}

It is known that $\mathrm{G}_{+}(k, n)$ defines a combinatorial polytope also known as 'Eulerian poset' [19]. We will verify one of the requirements for $\mathrm{OG}_{k}$ to be an Eulerian poset.

Eulerian poset. It is straightforward to count the number of cells at each level for arbitrary $k$. The result is most compactly summarized in terms of a generating function,

$$
T_{k}(q)=\sum_{l=0}^{k(k-1) / 2} T_{k, l} q^{l}
$$

$T_{k, l}$ is the number of on-shell diagrams with $2 k$ external legs and $l$ vertices without any bubble. Equivalently, $T_{k, l}$ is the number of $\mathrm{OG}_{k}$ tableaux at level $l$. A counting algorithm based on the construction of OG tableaux in section 4.1 can be easily implemented on a computer and generate $T_{k}(q)$. The results for $T_{k}(q)$ for small values of $k$ are given by

$$
\begin{aligned}
& T_{2}(q)=2+q, \\
& T_{3}(q)=5+6 q+3 q^{2}+q^{3}, \\
& T_{4}(q)=14+28 q+28 q^{2}+20 q^{3}+10 q^{4}+4 q^{5}+q^{6}, \\
& T_{5}(q)=42+120 q+180 q^{2}+195 q^{3}+165 q^{4}+117 q^{5}+70 q^{6}+35 q^{7}+15 q^{8}+5 q^{9}+q^{10} .
\end{aligned}
$$

After computing $T_{k}(q)$ up to $k=15$ using our own algorithm, we found that a beautiful closed-form expression for $T_{k}(q)$ had been known for decades, ${ }^{4,5}$

$$
T_{k}(q)=\frac{1}{(1-q)^{k}} \sum_{j=-k}^{k}(-1)^{j}\left(\begin{array}{c}
2 k \\
k+j
\end{array}\right) q^{j(j-1) / 2} .
$$

In special cases, this formula reproduces the simple general features discussed in section 4.1,

$$
T_{k}(1)=\frac{(2 k) !}{2^{k} k !}, \quad T_{k, 0}=\mathrm{C}_{k}, \quad T_{k, k(k-1) / 2}=1 .
$$

Another property of $T_{k}(q)$ that can be derived from (4.13) is that, for any $k$,

$$
T_{k}(-1)=\sum_{l}(-1)^{l} T_{k, l}=1 .
$$

We recognize this as one of the central properties of an Eulerian poset. In section 4.2, we assigned a coordinate patch of $\mathrm{POG}_{k}$ to each tableau. In this geometric context, $T_{k}(-1)$ is interpreted as the Euler characteristic of $\mathrm{POG}_{k}$. For $k=2,3$ (see figure 20), (4.14) matches 


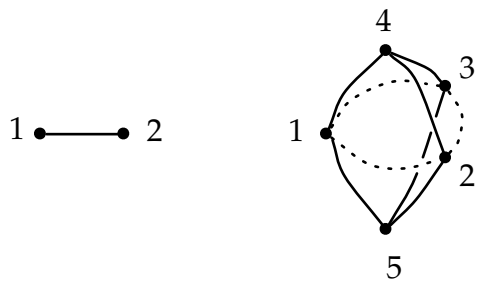

Figure 20. The ball topology of POG for $k=2,3$.

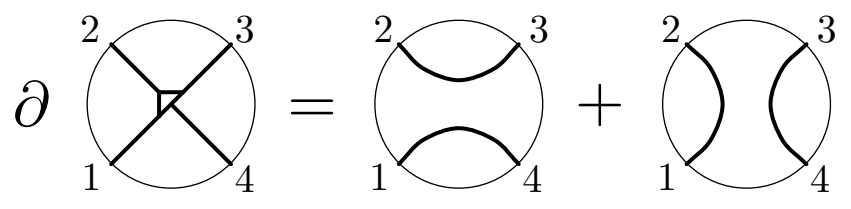

Figure 21. Schematics of the boundary operation.

with the fact that the POG has the topology of a ball. It remains to be seen whether the POG is a topological ball for all $k$.

Using the positroid stratification, we can refine the Eulerian poset structure. ${ }^{6}$ Consider the $k=2$ and $k=3$ examples in figure 12. We can compute an analogue of $T_{k}(q)$ for each chart containing several cells. They turn out to vanish in all charts, except for the smallest one with pivot columns $\{1,3, \cdots, 2 k-1\}$ which trivially gives 1 . We checked that the same phenomenon continues up to $k=7$, but have not attempted a proof for all $k$. Geometrically, it indicates that the subspaces of $\mathrm{POG}_{k}$ are likely to be topological balls. For instance, a level 2 cell for $k=3$ shown in figure 12 is topologically a square with four edges and four vertices.

Boundary operation. Following [7], we define the boundary operation $\partial$ acting on onshell diagrams such that it resolves each BCFW vertex in two ways shown in figure 21 . The corresponding $\mathrm{OG}_{2}$ tableaux (see figure 12) shows that the first term remains in the same coordinate chart as the original one, $\{1,2\}$, while the second term belongs to $\{1,3\}$. It is a general property of the canonical coordinate system; there always exist some boundaries that cannot be reached without changing coordinates.

One possible approach to put every boundary within reach in a single coordinate patch would be to use the cyclic gauge, where the odd-labelled columns form an identity matrix and the even-labelled columns form an $\mathrm{SO}(k)$ rotation matrix. This approach has its own drawbacks. First, the cyclic gauge necessarily introduces a BCFW bridge with non-adjacent source legs $\{1,3\}$ or $\{2,4\}$. As explained in [14], this type of BCFW bridge is substantially more complicated than the ones used in this paper. Second, positivity imposes coupled, non-linear relations among the angle variables of $\mathrm{SO}(k)$ rotation, in contrast to the simple

\footnotetext{
${ }^{4}$ See [36] and references therein.

${ }^{5} \mathrm{~A}$ similar result for $\mathrm{G}_{+}(k, n)$ was obtained in [37].

${ }^{6}$ We thank Yu-tin Huang for bringing [19] to our attention. See also [32].
} 


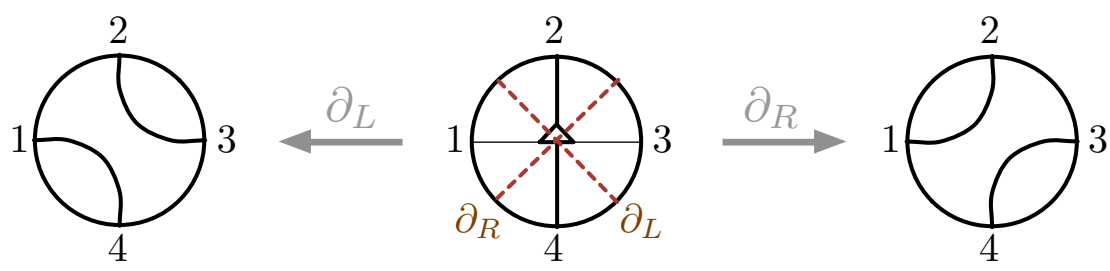

Figure 22. Boundary operation.

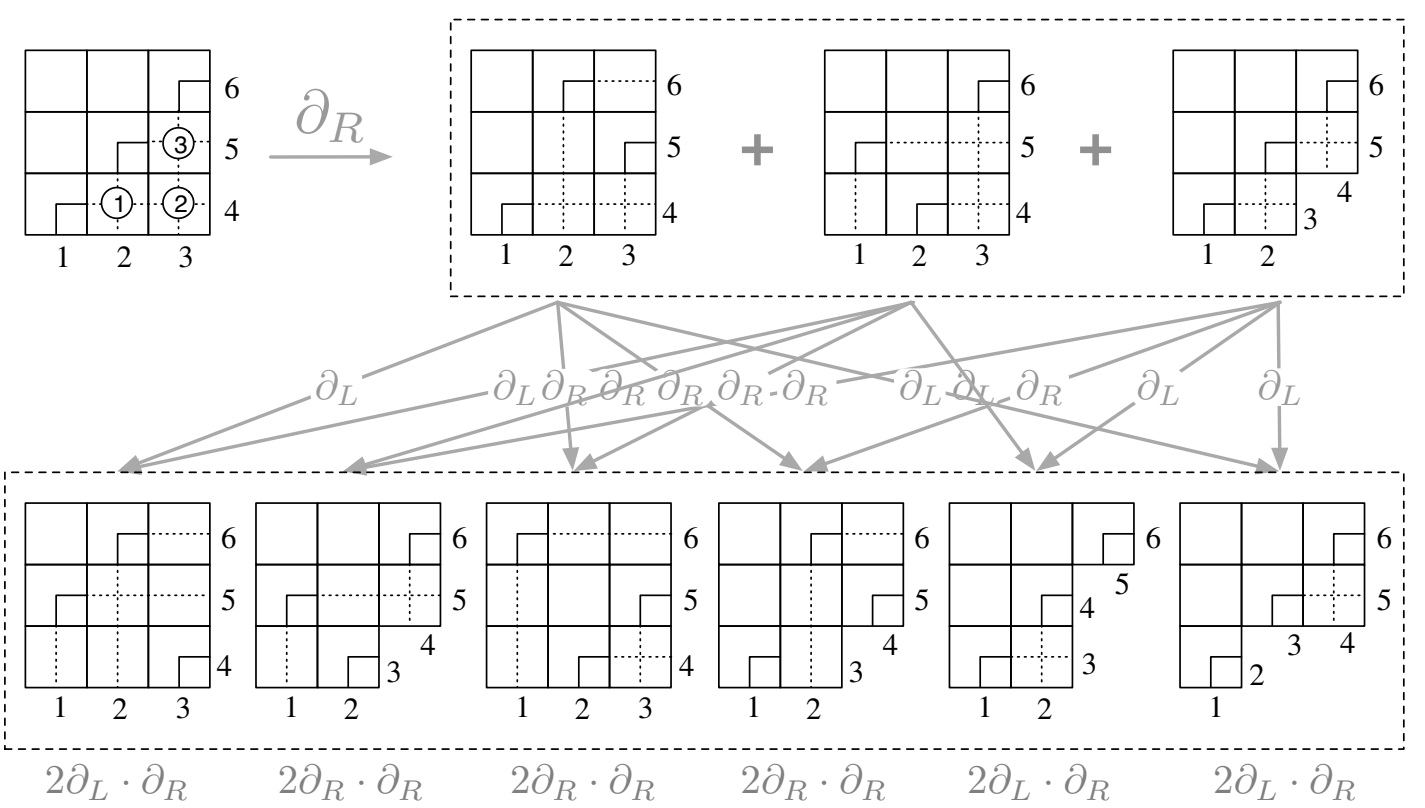

Figure 23. Boundary Operation $\partial^{2}=0(\bmod 2)$.

$t_{i} \geq 0$ conditions in this paper. For these reasons, we will stay within the canonical coordinate system and look for an alternative way to reach all boundaries.

It is convenient to separate the boundary operation $\partial$ into $\partial_{L}$ and $\partial_{R}$, according to the orientation of the resolved diagrams (see figure 22).

In [7], it was shown that $\partial^{2}=0(\bmod 2)$ holds for $\mathrm{G}_{+}(k, n)$ and conjectured that the $(\bmod 2)$ restriction could be dropped if suitable signs are attached to each on-shell diagram. ${ }^{7}$ Here, we will outline a similar argument for POG using an example without attempting a general proof. Applying $\partial$ to the $k=3$ top-cell, we first observe that $\partial_{L}$ results in bubble configurations, which we will discard by hand. Figure 23 shows not only that each diagram has two incoming arrows, implying $\partial^{2}=0(\bmod 2)$, but also that $\partial_{R}^{2}=0(\bmod 2)=\partial_{L} \cdot \partial_{R}$. In general, we have

$$
\partial_{L} \cdot \partial_{R}+\partial_{R} \cdot \partial_{L}=0(\bmod 2)=\partial_{R}^{2}=\partial_{L}^{2} .
$$

\footnotetext{
${ }^{7}$ This conjecture for $\mathrm{G}_{+}(k, n)$ is in fact known. It was shown in [17] that the poset for $\mathrm{G}_{+}(k, n)$ is a subposet of 'Bruhat order, and it is a classical result that the statement of the conjecture holds for Bruhat order. We thank T. Lam for explaining this point to us.
} 
Boundaries of a top-cell. The top-cell at each $k$ has precisely $k$ boundaries as can be seen in (4.11). We will identify them with the vanishing loci of the $k$ independent consecutive minors that appear in the original OG integral (2.8).

We begin with $k=3$ (see figure 23). In our coordinate system (4.7), the three independent consecutive minors are

$$
(123)=1, \quad(234)=\sinh t_{1} \sinh t_{2}, \quad(345)=\sinh t_{2} \sinh t_{3} .
$$

We can easily obtain these minors without constructing the whole $C$-matrix. The only relevant matrix elements for (234) and (345) are $c_{14}$ and $c_{36}$, respectively, which can be read off from the paths $1 \rightarrow 4$ and $3 \rightarrow 6$, respectively. These paths do not involve a turn so that each vertex contributes a $\sinh t_{i}$. Finally, the construction of POG guarantees that the overall sign should be positive.

A similar argument can be applied to consecutive minors for higher $k$. We simply summarize the results. First, $(123 \cdots k)=1=(k+1, \cdots, 2 k)$ by construction. For other consecutive minors, we can always draw a rectangle within the OG tableau whose right/bottom edges correspond to the sink/pivot columns participating in the minor. If we collect all BCFW variables inside the rectangle, the product of $\sinh t$ factors produces the correct result for the minor. For example, for a general $k$,

$$
(234 \cdots, k+1)=\prod_{i=1}^{k-1} \sinh t_{i}, \quad(345 \cdots k+2)=\prod_{i=2}^{2 k-3} \sinh t_{i}, \quad \text { and so on. }
$$

One may try to reach the boundaries of the top-cell by turning off some $t_{i}$. However, sometimes it forces two or more consecutive minors to vanish at the same time, leaving a bubble configuration. Among the $k(k-1) / 2$ BCFW variables, only $(k-1)$ of them can be safely turned off without generating a bubble. It is easy to see that they correspond to the $(k-1)$ vertices along the diagonal of the $\mathrm{OG}_{k}$ tableau.

By definition of the top-cell, the canonical coordinate does not allow $(123 \cdots k)$ to vanish. Recall from section 3.2 that, for $k=2$, the boundary with ' $(12)=0$ ' was reached by taking $t \rightarrow \infty$ in the canonical coordinates and performing a gauge transformation. A similar change of variable, which leads to a different coordinate patch, can reach the last boundary of a top-cell. In our prescription for computing the minors using rectangles, it is clear that all but $(123 \cdots k)$ contains $\sinh t_{k-1}$ coming from the lower right corner of the OG tableau for the top-cell. Taking $t_{k-1} \rightarrow \infty$ makes all consecutive minors except $(123 \cdots k)$ diverge. Since only the ratios between minors are gauge invariant, we may divide all minors by $\sinh t_{k-1}$ so that $(123 \cdots k)$ converges to zero and all other minors remain finite. This configuration is equal to the $\mathrm{OG}_{k}$ tableau whose the rightmost-bottom box is removed. Going back to the $k=3$ example, setting $t_{2} \rightarrow \infty$ and dividing all minors by $\sinh t_{2}$ gives

$$
(123)=0, \quad(234)=\sinh t_{1}, \quad(345)=\sinh t_{3} .
$$

This agrees with the result obtained from the (13)(25)(46) tableau in figure 18.

In summary, we have observed that $\mathrm{POG}_{k}$ is likely to form an Eulerian poset and to have a ball topolgy. As a partial attempt to state and prove these conjectures rigorously, 
we took an initial step to define the notion of boundary operation satifying $\partial^{2}=0$. Such $\partial$ would be naturally identified with the boundary operation of the usual simplicial homology on the geometric side. We verified that $\partial^{2}=0(\bmod 2)$ works for $\mathrm{POG}_{k}$ up to $k=3$ and is likely to generalize straightforwardly for higher $k$. But, dropping the 'modulo 2' restriction seems to be a difficult task. We hope to revisit these problems in a future work.

\section{Acknowledgments}

We are grateful to Saebyeok Jeong and Jihye Seo for collaboration at early stages of this work. We are also grateful to Yu-tin Huang for many enlightening discussions, helpful comments on the manuscript, and sharing a preliminary draft of [32] prior to publication. We thank Lauren Williams for pointing out errors in section 4.3 of the original version of this paper, and Thomas Lam for sharing the preprint [38] prior to publication and for many valuable comments. The work of JK is supported in part by the BK21 program of the Ministry of Education, Science and Technology of Korea, and the National Research Foundation of Korea (NRF) Grants 2010-0007512, 2012R1A1A2042474 and 2005-0049409 through the Center for Quantum Spacetime (CQUeST) of Sogang University. The work of SL is supported in part by the National Research Foundation of Korea (NRF) Grants 2012R1A1B3001085 and 2012R1A2A2A02046739.

Open Access. This article is distributed under the terms of the Creative Commons Attribution License (CC-BY 4.0), which permits any use, distribution and reproduction in any medium, provided the original author(s) and source are credited.

\section{References}

[1] H. Elvang and Y.-T. Huang, Scattering amplitudes, arXiv:1308.1697 [INSPIRE].

[2] N. Arkani-Hamed, F. Cachazo, C. Cheung and J. Kaplan, A duality for the $S$ matrix, JHEP 03 (2010) 020 [arXiv: 0907.5418] [INSPIRE].

[3] E. Witten, Perturbative gauge theory as a string theory in twistor space, Commun. Math. Phys. 252 (2004) 189 [hep-th/0312171] [INSPIRE].

[4] R. Roiban, M. Spradlin and A. Volovich, On the tree level S matrix of Yang-Mills theory, Phys. Rev. D 70 (2004) 026009 [hep-th/0403190] [InSPIRE].

[5] R. Britto, F. Cachazo and B. Feng, New recursion relations for tree amplitudes of gluons, Nucl. Phys. B 715 (2005) 499 [hep-th/0412308] [INSPIRE].

[6] R. Britto, F. Cachazo, B. Feng and E. Witten, Direct proof of tree-level recursion relation in Yang-Mills theory, Phys. Rev. Lett. 94 (2005) 181602 [hep-th/0501052] [INSPIRE].

[7] N. Arkani-Hamed et al., Scattering amplitudes and the positive Grassmannian, arXiv: 1212.5605 [INSPIRE].

[8] O. Aharony, O. Bergman, D.L. Jafferis and J. Maldacena, $\mathcal{N}=6$ superconformal Chern-Simons-matter theories, M2-branes and their gravity duals, JHEP 10 (2008) 091 [arXiv:0806.1218] [INSPIRE]. 
[9] K. Hosomichi, K.-M. Lee, S. Lee, S. Lee and J. Park, $\mathcal{N}=4$ superconformal Chern-Simons theories with hyper and twisted hyper multiplets, JHEP 07 (2008) 091 [arXiv:0805.3662] [INSPIRE].

[10] K. Hosomichi, K.-M. Lee, S. Lee, S. Lee and J. Park, $\mathcal{N}=5,6$ superconformal Chern-Simons theories and M2-branes on orbifolds, JHEP 09 (2008) 002 [arXiv:0806.4977] [INSPIRE].

[11] J. Bagger and N. Lambert, Three-algebras and $\mathcal{N}=6$ Chern-Simons gauge theories, Phys. Rev. D 79 (2009) 025002 [arXiv:0807.0163] [INSPIRE].

[12] M. Schnabl and Y. Tachikawa, Classification of $\mathcal{N}=6$ superconformal theories of ABJM type, JHEP 09 (2010) 103 [arXiv:0807.1102] [INSPIRE].

[13] S. Lee, Yangian invariant scattering amplitudes in supersymmetric Chern-Simons theory, Phys. Rev. Lett. 105 (2010) 151603 [arXiv:1007.4772] [INSPIRE].

[14] Y.-T. Huang and C. Wen, ABJM amplitudes and the positive orthogonal Grassmannian, JHEP 02 (2014) 104 [arXiv: 1309.3252] [INSPIRE].

[15] D. Gang, Y.-T. Huang, E. Koh, S. Lee and A.E. Lipstein, Tree-level recursion relation and dual superconformal symmetry of the ABJM theory, JHEP 03 (2011) 116 [arXiv:1012.5032] [INSPIRE].

[16] A. Postnikov, Total positivity, Grassmannians and networks, math.C0/0609764 [INSPIRE].

[17] A. Knutson, T. Lam and D. Speyer, Positroid varieties: juggling and geometry, arXiv: 1111.3660 .

[18] T. Lam and L.K. Williams, Total positivity for cominuscule Grassmannians, arXiv:0710.2932.

[19] L.K. Williams, Shelling totally nonnegative flag varieties, J. Reine Angew. Math. 609 (2007) 1 [math.RT/0509129].

[20] T. Bargheer, F. Loebbert and C. Meneghelli, Symmetries of tree-level scattering amplitudes in $\mathcal{N}=6$ superconformal Chern-Simons theory, Phys. Rev. D 82 (2010) 045016 [arXiv: 1003.6120] [INSPIRE].

[21] Y.-T. Huang and A.E. Lipstein, Amplitudes of $3 D$ and $6 D$ maximal superconformal theories in supertwistor space, JHEP 10 (2010) 007 [arXiv: 1004.4735] [INSPIRE].

[22] Y.-T. Huang and A.E. Lipstein, Dual superconformal symmetry of $\mathcal{N}=6$ Chern-Simons theory, JHEP 11 (2010) 076 [arXiv: 1008.0041] [INSPIRE].

[23] L.J. Mason and D. Skinner, Dual superconformal invariance, momentum twistors and Grassmannians, JHEP 11 (2009) 045 [arXiv:0909.0250] [INSPIRE].

[24] N. Arkani-Hamed, F. Cachazo and C. Cheung, The Grassmannian origin of dual superconformal invariance, JHEP 03 (2010) 036 [arXiv:0909.0483] [INSPIRE].

[25] N. Arkani-Hamed and J. Trnka, The amplituhedron, arXiv:1312.2007 [INSPIRE].

[26] J.L. Bourjaily, J. Trnka, A. Volovich and C. Wen, The Grassmannian and the twistor string: connecting all trees in $\mathcal{N}=4$ SYM, JHEP 01 (2011) 038 [arXiv:1006.1899] [INSPIRE].

[27] Y.-T. Huang and S. Lee, A new integral formula for supersymmetric scattering amplitudes in three dimensions, Phys. Rev. Lett. 109 (2012) 191601 [arXiv:1207.4851] [InSPIRE].

[28] F. Cachazo, S. He and E.Y. Yuan, Scattering in three dimensions from rational maps, JHEP 10 (2013) 141 [arXiv:1306.2962] [INSPIRE]. 
[29] O.T. Engelund and R. Roiban, A twistor string for the ABJ(M) theory, JHEP 06 (2014) 088 [arXiv: 1401.6242] [INSPIRE].

[30] S. Fomin and A. Zelevinsky, Cluster algebras. I. Foundations, J. Amer. Math. Soc. 15 (2002) 497 [math.RT/0104151].

[31] V.V. Fock and A.B. Goncharov, Moduli spaces of local systems and higher Teichmüller theory, Publ. Math. IHES 103 (2006) 1 [math.AG/0311149].

[32] Y.-T. Huang, C. Wen and D. Xie, The positive orthogonal Grassmannian and loop amplitudes of ABJM, arXiv:1402.1479 [INSPIRE].

[33] A. Agarwal, N. Beisert and T. McLoughlin, Scattering in mass-deformed $\mathcal{N} \geq 4$ Chern-Simons models, JHEP 06 (2009) 045 [arXiv:0812.3367] [INSPIRE].

[34] T. Bargheer, N. Beisert, F. Loebbert and T. McLoughlin, Conformal anomaly for amplitudes in $\mathcal{N}=6$ superconformal Chern-Simons theory, J. Phys. A 45 (2012) 475402 [arXiv: 1204.4406] [INSPIRE].

[35] Maclester journal of Catalan numbers, volume 1, http://www.macalester.edu/ abeverid/mjcn/mjcn2009.pdf, Saint Paul U.S.A. (2009).

[36] J. Riordan, The distribution of crossings of chords joining pairs of 2 points on a circle, Math. Comput. 29 (1975) 215.

[37] L.K. Williams, Enumeration of totally positive Grassmann cells, Adv. Math. 190 (2005) 319 [math.C0/0307271].

[38] T. Lam, The uncrossing partial order on matchings is Eulerian, arXiv:1406.5671. 